\title{
Sputum proteomics and airway cell transcripts of current and ex-smokers with severe asthma in U-BIOPRED: an exploratory analysis
}

\author{
Kentaro Takahashi ${ }^{1,2}$, Stelios Pavlidis ${ }^{3}$, Francois Ng Kee Kwong ${ }^{1}$, Uruj Hoda ${ }^{1}$, \\ Christos Rossios ${ }^{1}$, Kai Sun ${ }^{3}$, Matthew Loza ${ }^{4}$, Fred Baribaud ${ }^{4}$, Pascal Chanez ${ }^{5}$, \\ Steve J. Fowler ${ }^{6}$, Ildiko Horvath ${ }^{7}$, Paolo Montuschi ${ }^{8}$, Florian Singer ${ }^{9}$, \\ Jacek Musial ${ }^{10}$, Barbro Dahlen ${ }^{11}$, Sven-Eric Dahlen ${ }^{11}$, Norbert Krug ${ }^{12}$, \\ Thomas Sandstrom ${ }^{13}$, Dominic E. Shaw (1) ${ }^{14}$, Rene Lutter ${ }^{15}$, Per Bakke ${ }^{16}$, \\ Louise J. Fleming ${ }^{1}$, Peter H. Howarth ${ }^{17}$, Massimo Caruso (i) ${ }^{18}$, Ana R. Sousa ${ }^{19}$, \\ Julie Corfield ${ }^{20,21}$, Charles Auffray ${ }^{22}$, Bertrand De Meulder ${ }^{22}$, Diane Lefaudeux (1022, \\ Ratko Djukanovic ${ }^{17}$, Peter J. Sterk ${ }^{16}$, Yike Guo $^{3}$, lan M. Adcock ${ }^{1,3}$ and Kian \\ Fan Chung ${ }^{1,3}$, on behalf of the U-BIOPRED study group ${ }^{23}$
}

@ERSpublications

Inflammatory, oxidative/ER stress and epithelial barrier pathways are differentially activated in current smoking and ex-smoking severe asthma patients http://ow.ly/BVv530j3iP3

Cite this article as: Takahashi K, Pavlidis S, Ng Kee Kwong F, et al. Sputum proteomics and airway cell transcripts of current and ex-smokers with severe asthma in U-BIOPRED: an exploratory analysis. Eur Respir J 2018; 51: 1702173 [https://doi.org/10.1183/13993003.02173-2017].

ABSTRACT Severe asthma patients with a significant smoking history have airflow obstruction with reported neutrophilia. We hypothesise that multi-omic analysis will enable the definition of smoking and ex-smoking severe asthma molecular phenotypes.

The U-BIOPRED cohort of severe asthma patients, containing current-smokers (CSA), ex-smokers (ESA), nonsmokers and healthy nonsmokers was examined. Blood and sputum cell counts, fractional exhaled nitric oxide and spirometry were obtained. Exploratory proteomic analysis of sputum supernatants and transcriptomic analysis of bronchial brushings, biopsies and sputum cells was performed.

Colony-stimulating factor (CSF) 2 protein levels were increased in CSA sputum supernatants, with azurocidin 1, neutrophil elastase and CXCL8 upregulated in ESA. Phagocytosis and innate immune pathways were associated with neutrophilic inflammation in ESA. Gene set variation analysis of bronchial epithelial cell transcriptome from CSA showed enrichment of xenobiotic metabolism, oxidative stress and endoplasmic reticulum stress compared to other groups. CXCL5 and matrix metallopeptidase 12 genes were upregulated in ESA and the epithelial protective genes, mucin 2 and cystatin SN, were downregulated.

Despite little difference in clinical characteristics, CSA were distinguishable from ESA subjects at the sputum proteomic level, with CSA patients having increased CSF2 expression and ESA patients showing sustained loss of epithelial barrier processes.

The transcriptomic data have been deposited in the Gene Expression Omnibus database, www.ncbi.nlm.nih.gov/geo
(accession number GSE76225 for gene expression data of bronchial biopsies).

This aticle has supplenenty materia avalable from erjesjounats.com

Received: Dec 052017 | Accepted after revision: Feb 222018

Copyright @ERS 2018 
Affiliations: ${ }^{1}$ National Heart and Lung Institute, Imperial College London, and Biomedical Research Unit, Biomedical Research Unit, Royal Brompton and Harefield NHS Trust, London, UK. ${ }^{2}$ Research Centre for Allergy and Clinical Immunology, Asahi General Hospital, Matsudo, Japan. ${ }^{3}$ Dept of Computing and Data Science Institute, Imperial College London, London, UK. ${ }^{4}$ Janssen Research and Development, High Wycombe, UK. ${ }^{5}$ Assistance Publique des Hôpitaux de Marseille, Clinique des Bronches, Allergies et Sommeil, Aix Marseille Université, Marseille, France. ${ }^{6}$ Centre for Respiratory Medicine and Allergy, Institute of Inflammation and Repair, University of Manchester and University Hospital of South Manchester, Manchester Academic Health Sciences Centre, Manchester, UK. ${ }^{7}$ Semmelweis University, Budapest, Hungary. ${ }^{8}$ Università Cattolica del Sacro Cuore, Milan, Italy. ${ }^{9}$ Bern University Hospital, University of Bern, Bern, Switzerland. ${ }^{10}$ Dept of Medicine, Jagiellonian University Medical College, Krakow, Poland. ${ }^{11}$ Centre for Allergy Research, Karolinska Institutet, Stockholm, Sweden. ${ }^{12}$ Fraunhofer Institute for Toxicology and Experimental Medicine, Hannover, Germany. ${ }^{13}$ Dept of Public Health and Clinical Medicine, Umeå University, Umeå, Sweden. ${ }^{14}$ Respiratory Research Unit, University of Nottingham, Nottingham, UK. ${ }^{15}$ Academic Medical Centre, University of Amsterdam, Amsterdam, The Netherlands. ${ }^{16}$ Dept of Clinical Science, University of Bergen, Bergen, Norway. ${ }^{17}$ NIHR Southampton Respiratory Biomedical Research Unit, Clinical and Experimental Sciences and Human Development and Health, Southampton, UK. ${ }^{18}$ Dept Clinical and Experimental Medicine, University of Catania, Catania, Italy. ${ }^{19}$ Respiratory Therapeutic Unit, GSK, Stockley Park, UK. ${ }^{20}$ AstraZeneca R\&D, Molndal, Sweden. ${ }^{21}$ Areteva R\&D, Nottingham, UK. ${ }^{22}$ European Institute for Systems Biology and Medicine, CNRS-ENS-UCBL-INSERM, Lyon, France. ${ }^{23} \mathrm{~A}$ full list of the U-BIOPRED study group members can be found in the Acknowledgements section.

Correspondence: Kian Fan Chung, National Heart and Lung Institute, Imperial College London, Dovehouse Street, London, SW3 6LY, UK. E-mail: f.chungdimperial.ac.uk

\section{Introduction}

Severe asthma has been defined as asthma that requires treatment with high-dose inhaled corticosteroids and long-acting $\beta_{2}$-agonists and often systemic corticosteroids to prevent it from becoming "uncontrolled", or that remains "uncontrolled" despite this therapy [1]. A significant number of patients with asthma are current smokers or ex-smokers [2]. Asthmatic patients who smoke may develop poorly controlled asthma, a poor response to corticosteroid therapy, an accelerated decline in lung function and increased healthcare utilisation [3]. In an analysis of clinical phenotypes of severe asthma of the Unbiased Biomarkers for the Prediction of Respiratory Disease Outcomes (U-BIOPRED) cohort based on clinical and physiological features, a phenotype of severe asthma consisting of current and ex-smokers was characterised with late-onset asthma and moderate-to-severe chronic airflow obstruction [4]. This phenotype may represent an asthma-chronic obstructive pulmonary disease (COPD) overlap syndrome, with features of both diseases. In patients who were recruited as COPD patients in the COPDgene cohort, the patients who had a history of asthma before the age of 40 years and who had a smoking history of $\geqslant 10$ pack-years with spirometric evidence of severe airflow obstruction had more exacerbations, and a greater airway wall thickness on computed tomographic scans at all degrees of airflow obstruction compared to those with COPD alone [5]. This suggests that asthma may be driving airflow obstruction in concert with cigarette smoking exposure. The mechanisms underlying smoking-associated asthma is unclear but smoking-associated asthma has been considered as a non-T-helper type 2 (Th2) neutrophilic asthma [6].

The U-BIOPRED project recruited patients with severe asthma, including active smokers and ex-smokers [7]. One of the hallmarks of U-BIOPRED is the collection of omics data from blood, bronchial epithelium, bronchial biopsies and sputum cells, the analyses of which have yielded distinct molecular phenotypes of severe asthma $[8,9]$. In order to gain insight into the potential mechanisms that could underlie smoking or ex-smoking severe asthma, we examined the differential expression of genes and proteins in various compartments.

\section{Materials and methods Clinical data}

We enrolled 374 severe asthma patients in the U-BIOPRED cohort, divided into three groups by smoking status: current smokers with severe asthma (CSA), ex-smokers with severe asthma (ESA) and nonsmokers with severe asthma (NSA). We narrowed down the NSA to those who had never smoked (0 pack-years), although the original NSA group in the U-BIOPRED cohort contained patients whose smoking history was $<5$ pack-years. In addition, 81 nonsmoking healthy volunteers $(\mathrm{NH})$ with 0 pack-years were enrolled. Differential blood and induced sputum cell counts, serum total immunoglobulin E and skin prick tests, serum periostin and exhaled nitric oxide fraction $(\mathrm{FeNO})$ and pre- and post-bronchodilator spirometry were obtained $[8,9]$. Bronchial biopsies, bronchial brushings and sputum were obtained, as previously described [8]. Because of the bronchoscopy exclusion criteria (online supplementary material), only 95 bronchial brushings and 69 bronchial biopsies were obtained. The number of sputum samples for proteomic analysis was 88 . All subjects whose samples were adequate and underwent omics analyses are 
shown in online supplementary figure S1. The study was approved by the ethics committees of each of the 16 clinical recruiting centres. All subjects gave written and signed informed consent.

\section{Transcriptomic microarray analysis}

Sputum plugs were obtained and separated into cells and supernatants [7]. Cell pellets were used to prepare RNA using the miRNeasy mini kit (Qiagen, Valencia, CA, USA). Sputum samples with $>30 \%$ squamous cells were excluded from microarray analysis. Bronchial brushings and biopsy samples were immediately placed in TRIzol reagent (Invitrogen, ThermoFisher Scientific, Paisley, UK) and preserved at $-80^{\circ} \mathrm{C}$. Expression profiling of transcriptome was performed using GeneChip ${ }^{\circledR}$ Human Genome U133 Plus 2.0 Array (Affymetrix, Santa Clara, CA, USA) as previously described [8, 9]. Pathway analysis, enrichment analysis and functional clustering of differentially expressed genes (DEGs) were performed as described previously $[8,9]$ and protein interaction analysis using annotated protein-coding genes was performed using STRING (version 10.0; STRING Consortium 2016, www.string-db.org) [10].

\section{SomaLogic proteomic technique}

The SOMAscan proteomic assay of sputum supernatants performed by SomaLogic (Boulder, CO, USA) was used [9].

\section{Gene set variation analysis}

Gene set variation analysis (GSVA) was performed in $\mathrm{R}$ using the Bioconductor GSVA package for estimating variation of gene set enrichment [11]. Gene sets were obtained from the Molecular Signatures Database v5.2 (MSigDB) (http://software.broadinstitute.org/gsea/msigdb) or from published papers (online supplementary table S1). We used Immunomap graphics (Johnson \& Johnson, New Brunswick, NJ, USA) for visualisation.

\section{Statistical analysis}

All datasets were quality controlled and normalised, followed by adjustment of batch effects using ComBat tools and uploaded into tranSMART, an open-source knowledge management platform for sharing research data supported by European Translational Information and Knowledge Management Services (eTRIKS) [8, 9]. All categorical variables were analysed using Fisher's exact test. Continuous variables were analysed using Kruskal-Wallis testing. Gene and protein expression data were analysed using multivariate ANOVA; age, sex and systemic corticosteroids use were analysed as covariates. A p-value $<0.05$ was considered significant. A linear model for microarray data (Bioconductor limma package for R) with Benjamini-Hochberg false discovery rate (FDR) correction was used in the analysis of the DEGs and for GSVA. Fold change $\geqslant 1.5$ and FDR $<0.05$ was considered statistically significant in transcriptomic and proteomic analyses. When using GSVA, FDR $<0.05$ was considered statistically significant. Statistical analyses were performed using $\mathrm{R}$ version 3.3.1 (R Core Team, 2016; www.r-project.org).

\section{Results}

Clinical characteristics of subjects with sputum SomaLogic data

Table 1 shows the characteristics of subjects who provided sputum samples for SomaLogic analysis. FeNO levels of CSA subjects were lower than in the other severe asthma groups. Although there were numerical differences in blood eosinophil counts (CSA 259 cells $\mu \mathrm{L}^{-1}$, ESA 296 cells $\mu \mathrm{L}^{-1}$ and NSA 407 cells $\mu \mathrm{L}^{-1}$ ), sputum eosinophils (CSA 7.2\%, ESA $14.8 \%$ and NSA 18.8\%) and the proportion of those on oral corticosteroids (CSA 30.0\%, ESA $63.6 \%$ and NSA 45.7\%), these were not statistically significant. NSA patients had the highest blood and sputum eosinophil counts. No differences were seen between the three severe asthma groups in terms of pulmonary function, airway reversibility, clinical (asthma control test (ACQ)-7)) and quality of life (asthma quality of life questionnaire (AQLQ)) measures or in exacerbations in the previous year.

\section{Comparison of differentially expressed proteins}

Sputum SomaLogic analysis adjusted for age, sex and systemic corticosteroid use identified 13, 63 and 42 differentially expressed proteins (DEPs) between CSA and $\mathrm{NH}$, ESA and $\mathrm{NH}$, and NSA and $\mathrm{NH}$, respectively (figure $1 \mathrm{a}-\mathrm{c}$ ). The DEPs are shown in figure $1 \mathrm{~d}$ and online supplementary file 1 . Only five proteins distinguished CSA-NH from NSA-NH, including colony stimulating factor (CSF)2, also known as granulocyte-macrophage colony-stimulating factor, CXCL8/interleukin (IL)-8 and anterior gradient protein (AGR)2 (table 2). CXCL8 did not distinguish between the CSA-NH and ESA-NH groups. CSF2 is critical for the proliferation, differentiation and survival of granulocytes, monocytes and macrophages [12], whereas AGR2 is involved in mucin 5AC (MUC5AC) production by asthmatic epithelial cells [13]. Sputum levels of CSF2 and AGR2 and the sputum gene expression of MUC5AC were highest in CSA 
TABLE 1 Patient characteristics for sputum SomaLogic analyses

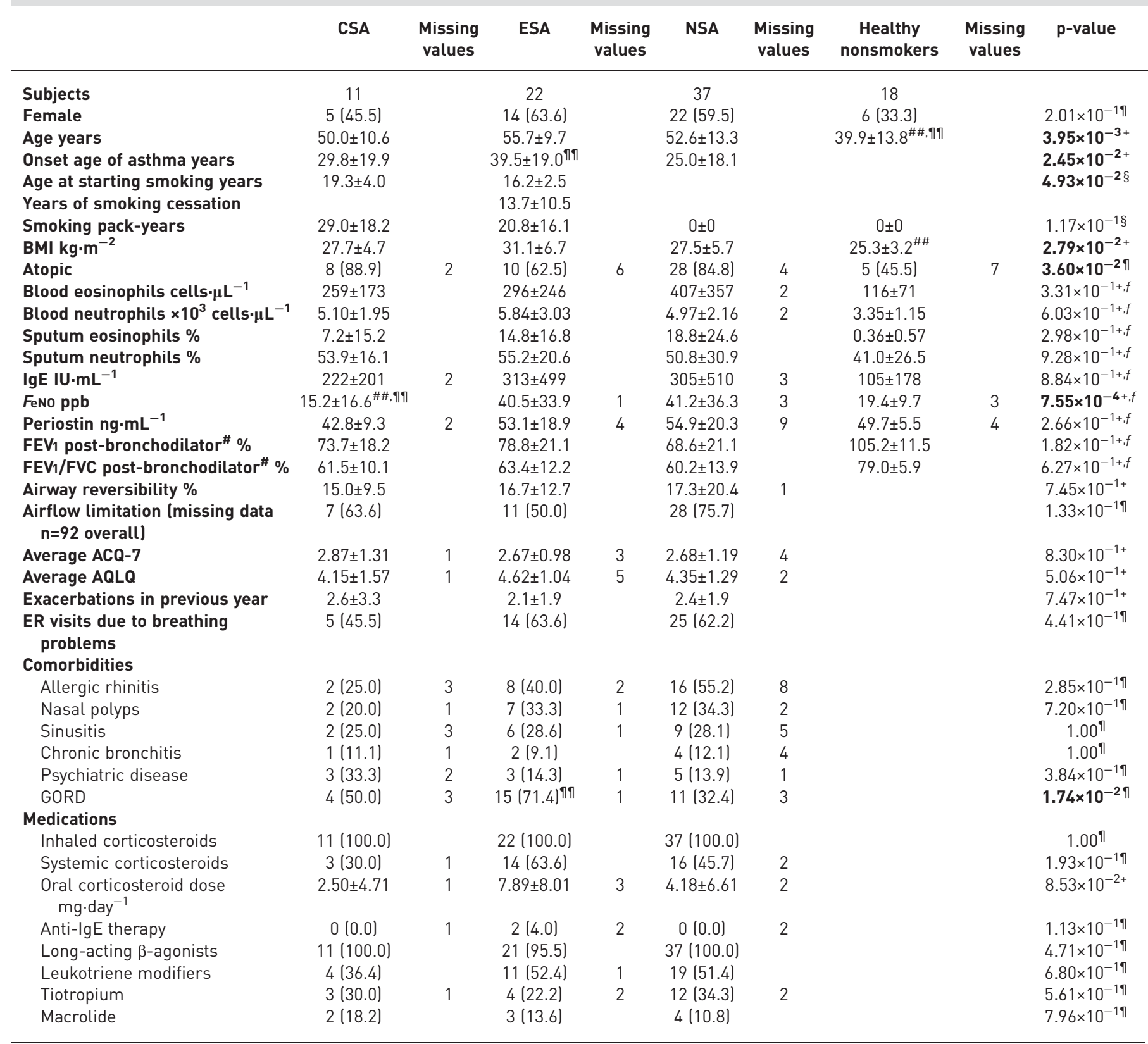

Data are presented as $n, n(\%)$ or mean $\pm S D$, unless otherwise stated. Bold type represents statistical significance ( $<<0.05)$. CSA: severe asthma current smokers; ESA: severe asthma ex-smokers; NSA: severe asthma nonsmokers; BMI: body mass index; Ig: immunoglobulin; Feno: fractional exhaled nitric oxide; FEV1: forced expiratory volume in $1 \mathrm{~s}$; FVC: forced vital capacity; ACQ: asthma control questionnaire; AQLQ: asthma quality of life questionnaire; ER: emergency room; GORD: gastro-oesophageal reflux disease. " : spirometry data without bronchodilator were used for healthy subjects; statistical analysis was performed using ๆ: Fisher's exact test, ${ }^{+}$: Kruskal-Wallis test; or ${ }^{\S}$ : Mann-Whitney U-test; ${ }^{f}$ : healthy subjects were excluded from statistical analyses of several items; ${ }^{\# \#}$ : $p<0.05$ versus ESA; ${ }^{\text {กा? }}$ : $p<0.052$ versus NSA.

patients (figure $2 \mathrm{a}$ and $\mathrm{b}$ and online supplementary figure S2). This suggests that CSA is associated with macrophage/neutrophil recruitment and mucus production.

34 DEPs distinguished ESA-NH from NSA-NH and included azurocidin (AZU)1, neutrophil elastase (ELANE), complement factor properdin (CFP) and C-X-C motif chemokine ligand (CXCL)8 (table 3, figure $2 \mathrm{c}-\mathrm{f}$ ). AZU1 possesses monocyte chemotactic and antimicrobial activity [14] and CFP positively regulates the alternative complement system [15]. 29 proteins overlapped between ESA-NH and NSA-NH, including C-reactive protein, CSF1 receptor, inducible T-cell costimulatory ligand, FCGR2A and catalase (CAT) (table 3, figure 1d). In contrast, there were 13 DEPs, including protein disulfide isomerase family A 
- Fold change $\geqslant 2.0$. Fold change $\leqslant 2.0$
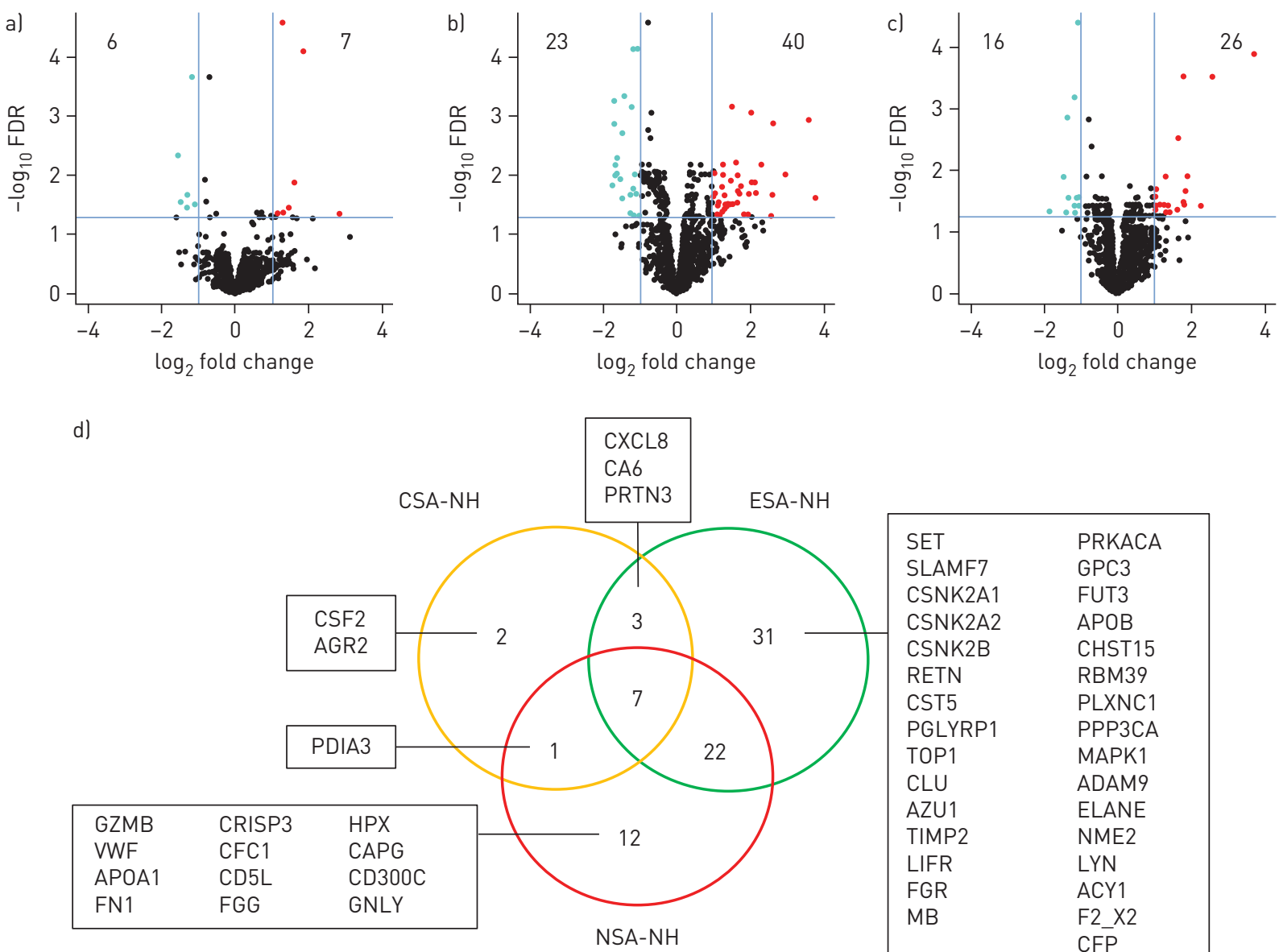

\begin{tabular}{|ll|}
\hline SET & PRKACA \\
SLAMF7 & GPC3 \\
CSNK2A1 & FUT3 \\
CSNK2A2 & APOB \\
CSNK2B & CHST15 \\
RETN & RBM39 \\
CST5 & PLXNC1 \\
PGLYRP1 & PPP3CA \\
TOP1 & MAPK1 \\
CLU & ADAM9 \\
AZU1 & ELANE \\
TIMP2 & NME2 \\
LIFR & LYN \\
FGR & ACY1 \\
MB & F2_X2 \\
& CFP \\
\hline
\end{tabular}

FIGURE 1 Phenotypic differences among severe asthma current smokers (CSA), severe asthma ex-smokers (ESA) and severe asthma nonsmokers (NSA) were unveiled using the SomaLogic linear model for microarray (limma) analysis of sputum. a-c) Volcano plots showing differentially expressed proteins (DEPs) in limma of sputum SomaLogic in the following comparisons: a) CSA and healthy nonsmokers (NH); b) ESA and NH; and c) NSA and NH. The proteins whose absolute fold change $\geqslant 2.0$ at false discovery rate (FDR) $<0.05$ were regarded as DEPs. The number of DEPs of each comparison is shown in the left and right upper areas of each plot. d) Venn diagram showing the numbers and names of DEPs in each comparison.

member 3, granzyme B (GZMB) and CD5 antigen-like (CD5L) (table 3, figure 1d). GZMB is a cytoplasmic granule of cytotoxic T-cells and natural killer cells, and is involved in apoptosis, chronic inflammation and wound healing [16]. CD5L, expressed in lymphoid tissues, lung epithelial cells or tissue macrophages, plays multiple roles in inflammation, such as promoting macrophage phagocytosis [17].

In summary, while CSA was associated with proteins involved in macrophage recruitment and mucus production and both ESA and NSA were associated with proteins with inflammatory and immune responses characterised by T-cell-mediated acquired immunity in common, proteins linked to neutrophilic activity were more closely related to ESA than to other groups. However, this was not reflected in a significant difference in sputum neutrophilia in these subjects (table 1). In addition, the protein expression of CAT, a key antioxidant, was upregulated equally in all severe asthma groups compared with $\mathrm{NH}$ (figure $2 \mathrm{~g}$ ).

\section{Pathway analysis of DEPs}

Pathway analysis of sputum DEPs indicated that ESA-NH was associated with phagocytosis, response to chemicals, response to multicellular organisms, chemotaxis, myeloid cell differentiation and innate immunity and inflammation, while NSA-NH was associated with acute-phase inflammation, platelet degranulation, response to wounding and the immune system (online supplementary table S2). Overall, different pathways were activated between CSA and NSA and airway epithelial damage may be associated with ESA. 
TABLE 2 Differentially expressed proteins between severe asthma current smokers (CSA) and healthy nonsmokers (NH) and severe asthma nonsmokers (NSA) and NH by sputum assay

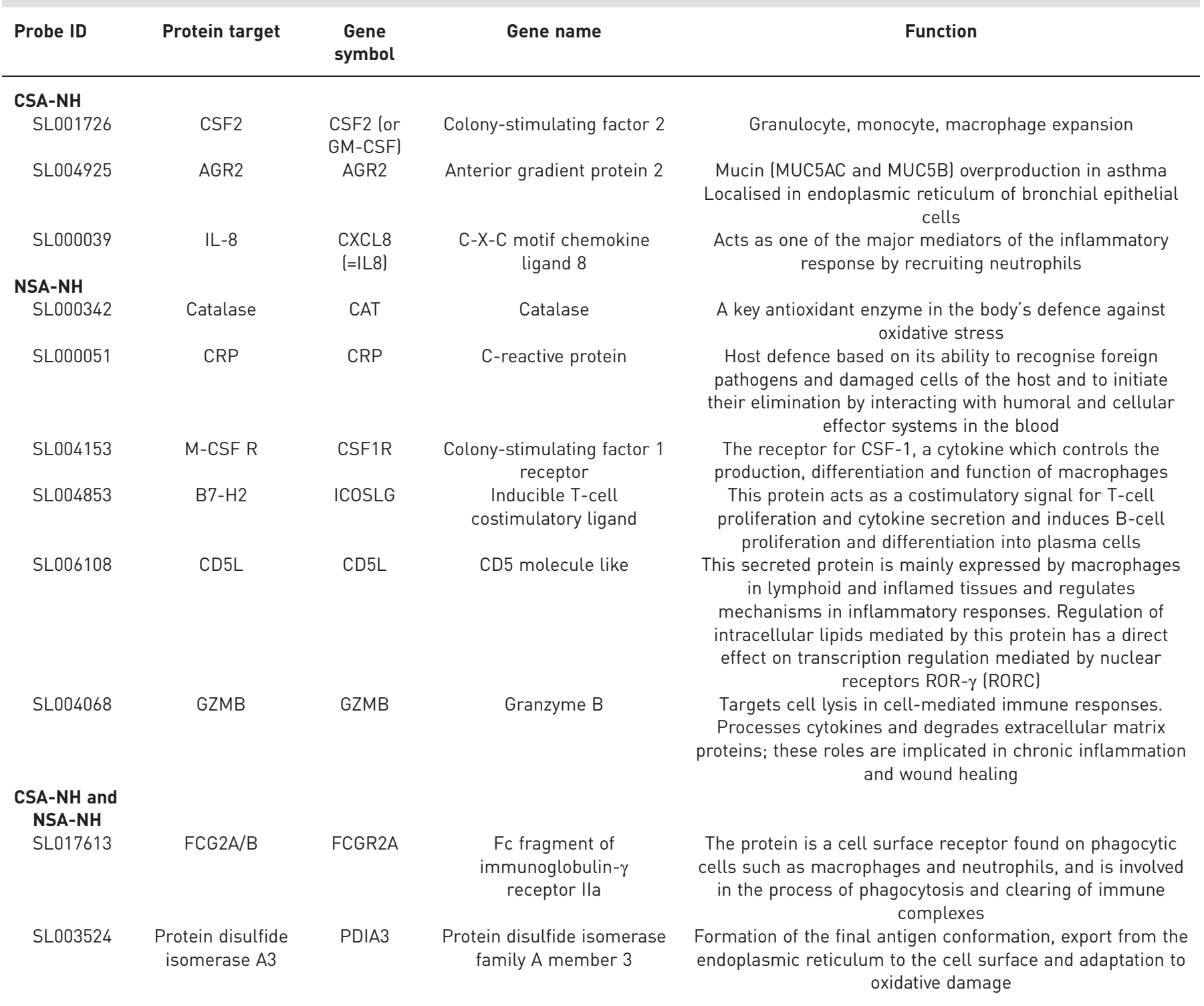

Assay performed by SomaLogic (Boulder, CO, USA). GM-CSF: granulocyte-macrophage colony-stimulating factor; IL: interleukin.

Characteristics of patients with transcriptomic analysis in bronchial biopsies and brushings

We found increased blood neutrophils and lower FeNO levels in CSA compared to NSA subjects providing bronchial brushings and biopsies for analysis, although the proportion of patients who took systemic corticosteroids or the dose of oral corticosteroids was no different between the two severe asthma groups (online supplementary tables S3-S5). There were no significant differences in blood eosinophil, sputum eosinophil and sputum neutrophil counts, and in pulmonary function, ACQ-7, AQLQ or the number of exacerbations in the previous year among the three severe asthma groups. The subjects who provided samples for sputum transcriptomics did not completely overlap with those providing sputum proteomics, but the clinical characteristics were similar (online supplementary table S5).

\section{DEGs between CSA and NSA}

We detected 142 significant DEG probes in bronchial brushings, 23 in bronchial biopsies and 15 in sputum samples between CSA and NSA (figure $3 \mathrm{a}-\mathrm{c}$ and online supplementary file 2). There were no significant DEG probes (FDR $>0.05$ ) in any samples between ESA and NSA (online supplementary file 3 ). Hierarchical clustering of the 142 DEGs from bronchial brushings indicated that although CSA and NSA were clearly distinct, NSA and ESA did not cluster separately (figure 3d). 

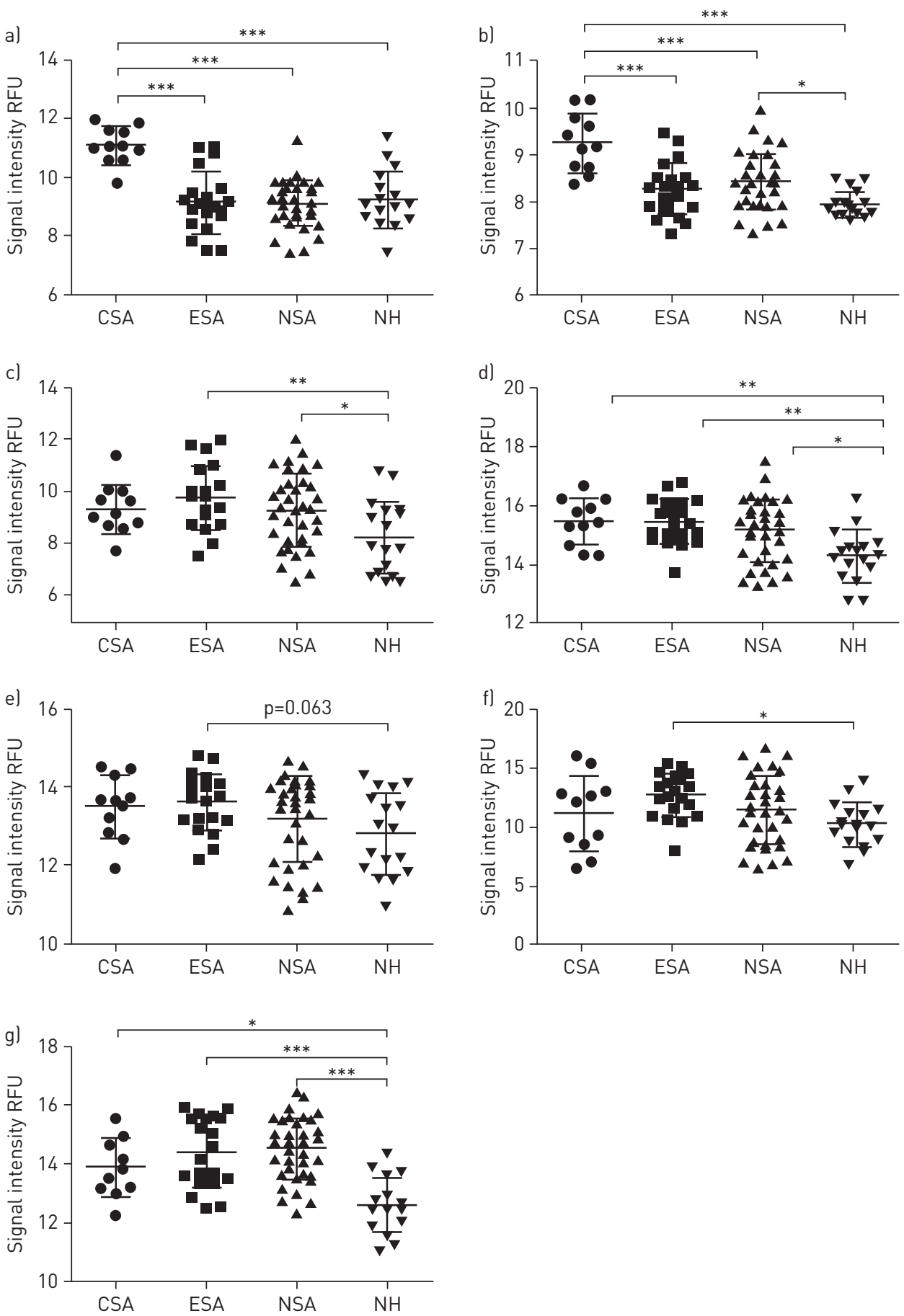

FIGURE 2 Differentially expressed proteins in severe asthma sputum according to smoking status. Dot plots with mean $\pm S D$ showing signal intensity levels of protein expression of a) colony-stimulating factor (CSF)-2, b) anterior gradient protein (AGR)2, c) azurocidin (AZU)1, d) C-X-C motif chemokine ligand (CXCL)8, e) neutrophil elastase (ELANE), f) complement factor properdin (CFP) and g) catalase (CAT) in sputum by SomaLogic (Boulder, CO, USA) analysis in severe asthma current smokers (CSA), severe asthma ex-smokers (ESA), severe asthma nonsmokers (NSA) and healthy nonsmokers (NH). RFU: relative fluorescence units, *: $p<0.05 ;{ }^{* *}: p<0.01 ;{ }^{* * *}: p<0.001$.

The DEGs between CSA and NSA are implicated in oxidation-reduction, xenobiotic metabolism and endoplasmic reticulum stress (online supplementary file 2). Cytochrome P450 family 1 subfamily B member 1 (CYP1B1) and aldehyde dehydrogenase 3 family member A1 (ALDH3A1), which were overexpressed in bronchial brushings of CSA compared to other groups (figure $4 \mathrm{a}$ and b) play a role in 
TABLE 3 Differentially expressed key proteins in sputum SomaLogic analysis comparing severe asthma ex-smokers (ESA) and healthy nonsmokers (NH) with severe asthma nonsmokers (NSA) and $\mathrm{NH}$

Probe ID Protein target $\begin{gathered}\text { Gene } \\ \text { symbol }\end{gathered} \quad$ Gene name $\quad$ Function

\section{ESA-NH}

SL004589

SL000401

SL003192

SL000039

NSA-NH

SL003524

SL006108

SL004068

\section{ESA-NH and}

NSA-NH

SL017613

SL000342

SL000051

SL004153

SL004853
GZMB

GZMB

Granzyme B

M-CSF R

CSF1R

AZU1

ELANE

Neutrophil elastase

Complement factor properdin

C-X-C motif chemokine ligand 8

$(=\mid L-8)$

PDIA3

CD5L

$\begin{array}{cr}\text { FCG2A/B } & \text { FCGR2A } \\ \text { Catalase } & \text { CAT } \\ \text { CRP } & \text { CRP } \\ \text { M-CSF R } & \text { CSF1R } \\ \text { B7-H2 } & \text { ICOSLG }\end{array}$

Colony-stimulating factor 1 receptor Inducible T-cell costimulatory ligand
A pre-proprotein of a mature azurophil granule antibiotic protein with monocyte chemotactic and antimicrobial activity

This protease hydrolyses proteins within specialised neutrophil lysosomes, called azurophil granules, as well as proteins of the extracellular matrix

A positive regulator of the alternative pathway of complement system

Acts as one of the major mediators of the inflammatory response by recruiting neutrophils

Formation of the final antigen conformation, export from the endoplasmic reticulum to the cell surface and adaptation to oxidative damage

This secreted protein is mainly expressed by macrophages in lymphoid and inflamed tissues and regulates mechanisms in inflammatory responses. Regulation of intracellular lipids mediated by this protein has a direct effect on transcription regulation mediated by nuclear receptors ROR- $\gamma$ (RORC)

Targets cell lysis in cell-mediated immune responses. This protein also processes cytokines and degrades extracellular matrix proteins, and these roles are implicated in chronic inflammation and wound healing

Cell surface receptor found on phagocytic cells such as macrophages and neutrophils, is involved in the process of phagocytosis and clearing of immune complexes

A key antioxidant enzyme in the body's defence against oxidative stress

Host defence based on its ability to recognise foreign pathogens and damaged cells of the host and to initiate their elimination by interacting with humoral and cellular effector systems in the blood

The receptor for CSF1, a cytokine which controls the production, differentiation and function of macrophages

This protein acts as a costimulatory signal for T-cell proliferation and cytokine secretion and induces B-cell proliferation and differentiation into plasma cells

Assay performed by SomaLogic (Boulder, CO, USA). IL: interleukin.

metabolising polycyclic aromatic hydrocarbons or aldehydes [18]. The oxidative stress genes, NAD(P)H quinone dehydrogenase 1 (NQO1) and aldo-keto reductase family 1 member C1 (AKR1C1), were also highly expressed in CSA bronchial brushings (figure $4 \mathrm{c}$ and $\mathrm{d}$ ). The endoplasmic reticulum plays a central role in the protein biosynthesis, correct protein folding and post-transcriptional modifications [19]. Accumulation of unfolded and misfolded proteins, termed endoplasmic reticulum stress, leads to the unfolded protein response and inflammation [20]. Heat shock protein family A (Hsp70) member 5 (HSPA5), a key mediator of endoplasmic reticulum stress, was significantly upregulated in CSA compared to NSA in bronchial brushings and biopsies (figure 4e).

Pathway analysis using DEGs between CSA and NSA

Pathway analysis indicated that oxidation-reduction, chemical metabolism and endoplasmic reticulum stress were different between CSA and NSA (online supplementary tables S6 and S7). These results suggest that the lung epithelial cells of CSA patients are under more potent chemical, oxidative and endoplasmic reticulum stresses than those of NSA patients. 


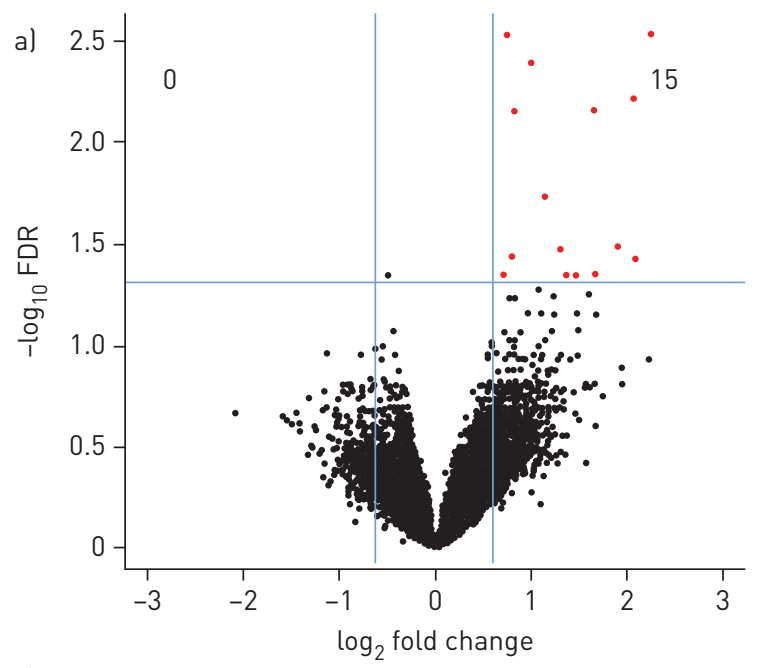

c)

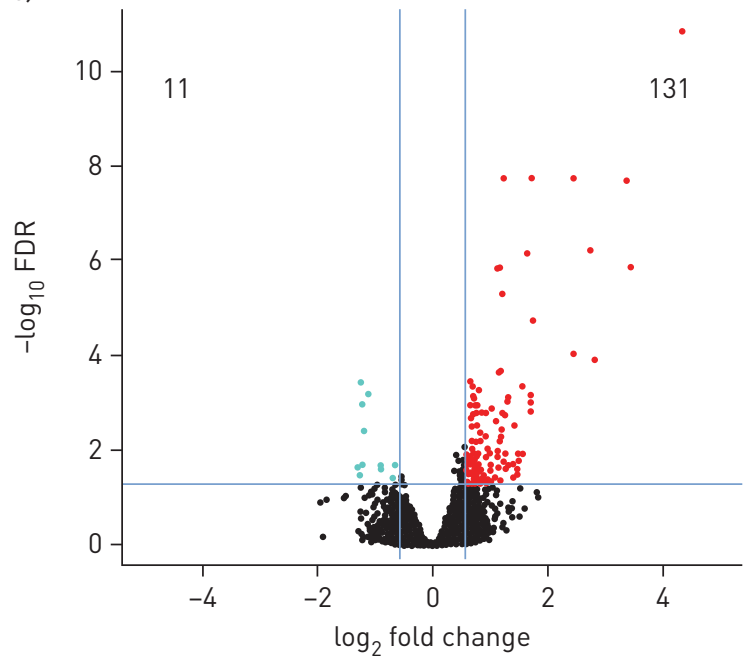

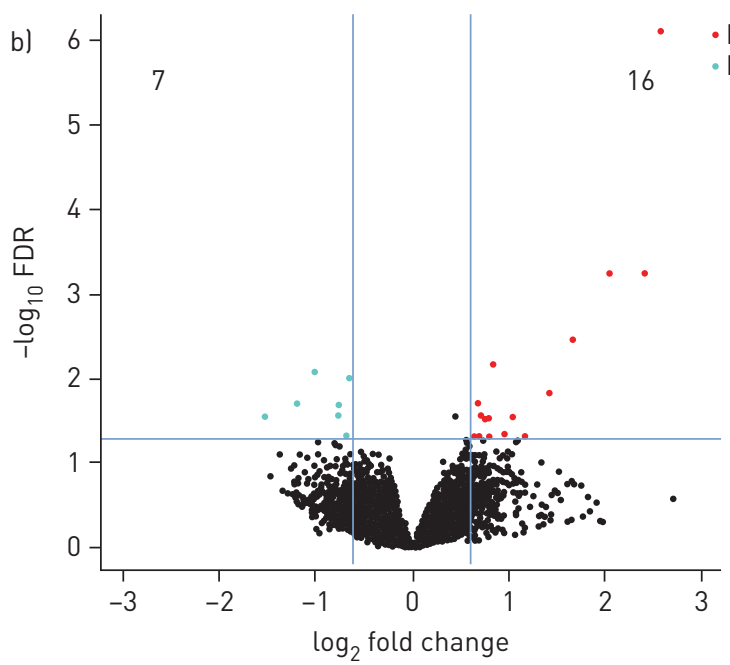

d)
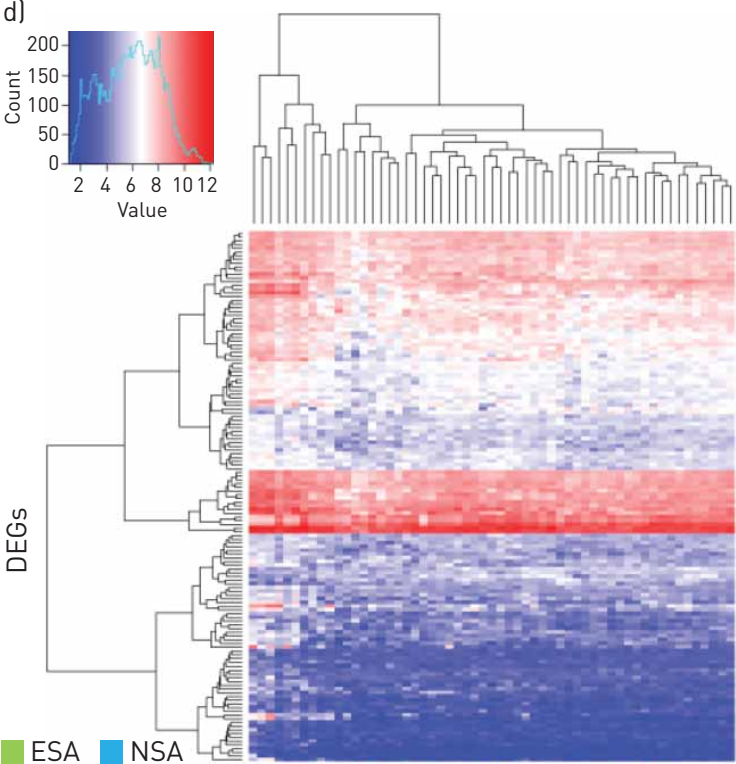

Low gene expression

High gene expression

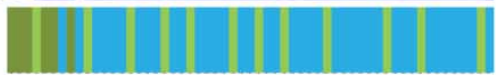

FIGURE 3 Differentially expressed genes (DEGs) in current smokers (CSA) and nonsmokers (NSA) with severe asthma. Volcano plots showing DEGs between CSA and NSA in a) sputa, b) bronchial biopsies and c) bronchial brushings. The genes whose absolute fold change (FC) $\geqslant 1.5$ at a false discovery rate (FDR) $<0.05$ are shown as coloured dots. The number of DEGs in each sample is shown in the left and right upper areas of each plot. d) Hierarchical clustering for DEGs from bronchial brushings in severe asthma patients. ESA: severe asthma ex-smokers.

GSVA of bronchial brushings and biopsies

GSVA confirmed the selective enrichment of xenobiotic metabolism by cytochrome P450, glutathione metabolism, response to oxidative stress, endoplasmic reticulum stress, unfolded protein response, lysosome or glycolysis and gluconeogenesis pathways in bronchial brushings (figure 5a-g) and biopsies (figure 6a-g) in the CSA group. There were no significant differences between ESA and NSA for these pathways. Using the signatures for active smoking obtained from SPIRA et al. [21], we confirmed that bronchial brushings and biopsies from CSA were enriched for the active smoking-related gene and that both CSA and ESA were enriched for the pack-year signature (online supplementary table S1 and figure S3).

DEGs in sputum, bronchial biopsies and epithelial brushings

As we could not detect any DEGs between ESA and NSA at the FDR $<0.05$ level, we undertook a discovery approach using a less stringent analysis strategy. Genes whose absolute fold change was $\geqslant 2.0$ in limma were used to clarify the phenotypic difference between ESA and NSA (online supplementary file 2). 27 genes (35 probes) were upregulated in ESA sputum samples including matrix metallopeptidase (MMP) 
a)

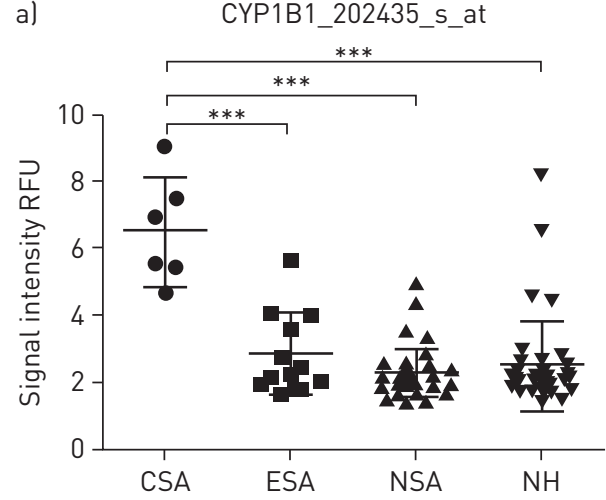

c)

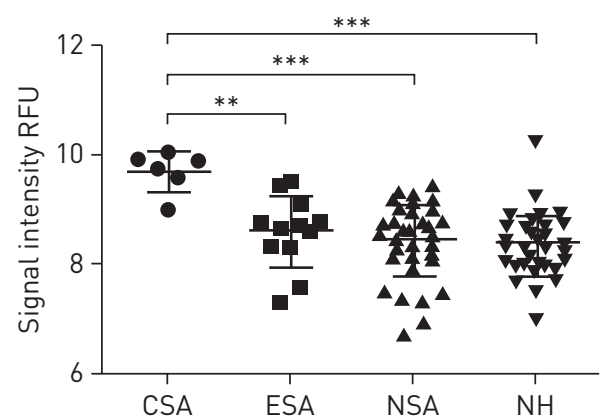

e)

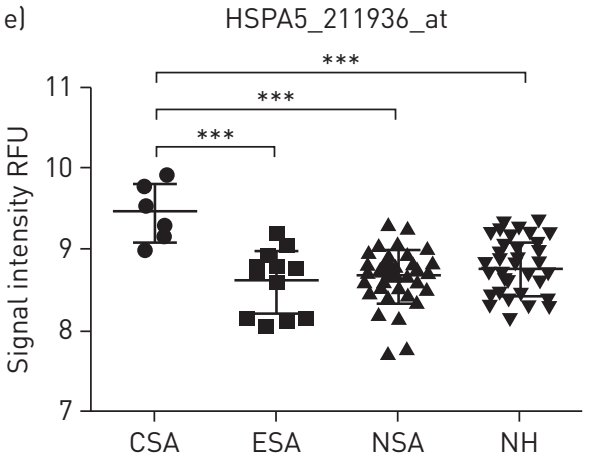

b)

ALDH3A1_205623_at

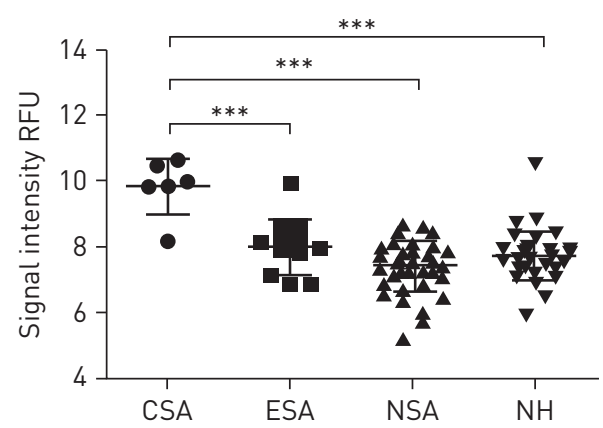

d)

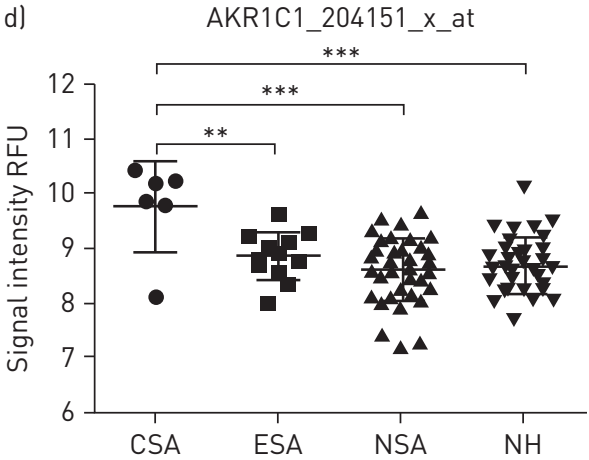

FIGURE 4 Differentially expressed genes (DEGs) associated with metabolism of xenobiotics, oxidative stress and endoplasmic reticulum stress in bronchial brushings. Dot plots showing DEGs in bronchial brushings associated with a) xenobiotic metabolism by cytochrome P450 family 1 subfamily B member 1 (CYP1B1), b) aldehyde dehydrogenase 3 family member $A 1$ (ALDH3A1), c) NAD(P)H quinone dehydrogenase 1 (NQ01), d) aldo-keto reductase family 1 member $\mathrm{C} 1$ (AKR1C1) and e) heat shock protein family A (Hsp70) member 5 (HSPA5). RFU: relative fluorescence units; CSA: severe asthma current smokers; ESA: severe asthma ex-smokers; NSA: severe asthma nonsmokers; NH: healthy nonsmokers. ${ }^{* *}: p<0.01{ }^{* * *}: p<0.001$.

12, neuropilin (NRP)1, Toll-interleukin 1 receptor domain containing adaptor protein (TIRAP), CXCL5 and pro-platelet basic protein (PPBP) (online supplementary table S8). MMP12 has been associated with decreased lung function and COPD, TIRAP is involved in the Toll-like receptor (TLR) signalling pathway and both CXCL5/ENA-78 and PPBP/CXCL7 are potent neutrophil chemoattractants and activators [22]. Innate immunity, including the complement system, TLR signalling and neutrophilic inflammation may be characteristics of ESA.

Six downregulated DEGs (fold change $\leqslant 0.5$ ) distinguished ESA from NSA in bronchial brushings, namely carboxypeptidase (CP)A3, cystatin SN (CST1), immunoglobulin- $\kappa$ constant (IGKC), mucin 2, oligomeric mucus/gel-forming (MUC2) and tryptase $\alpha / \beta 1$ (TPSAB1) (online supplementary table S8). CPA3 and TPSAB1 are mast cell biomarkers and are found to be elevated in asthma patients [23]. CST1 is a cysteine proteinase inhibitor that has a protective effect on epithelium [24]. MUC2 provides a protective barrier for airways against particles or infectious agents [25]. This suggests that ESA subjects have a lesser protective epithelial barrier and reduced mast cell activity compared with NSA. 
a)
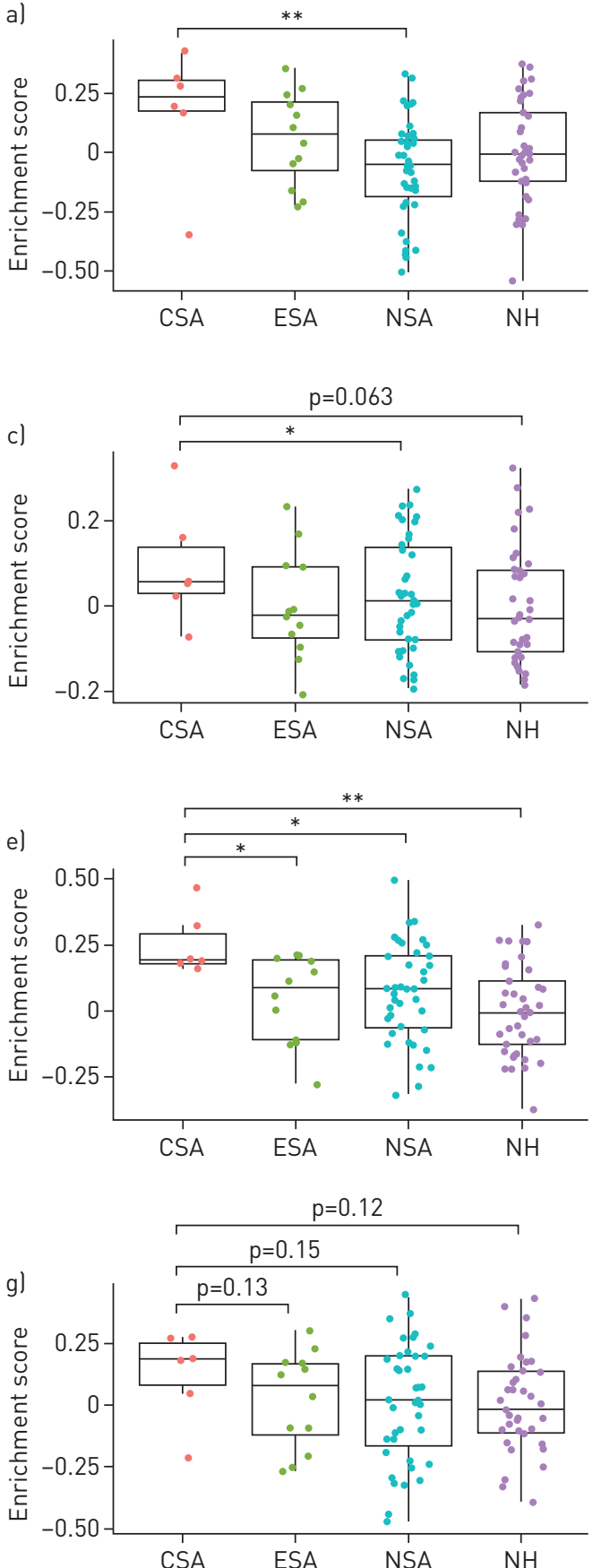
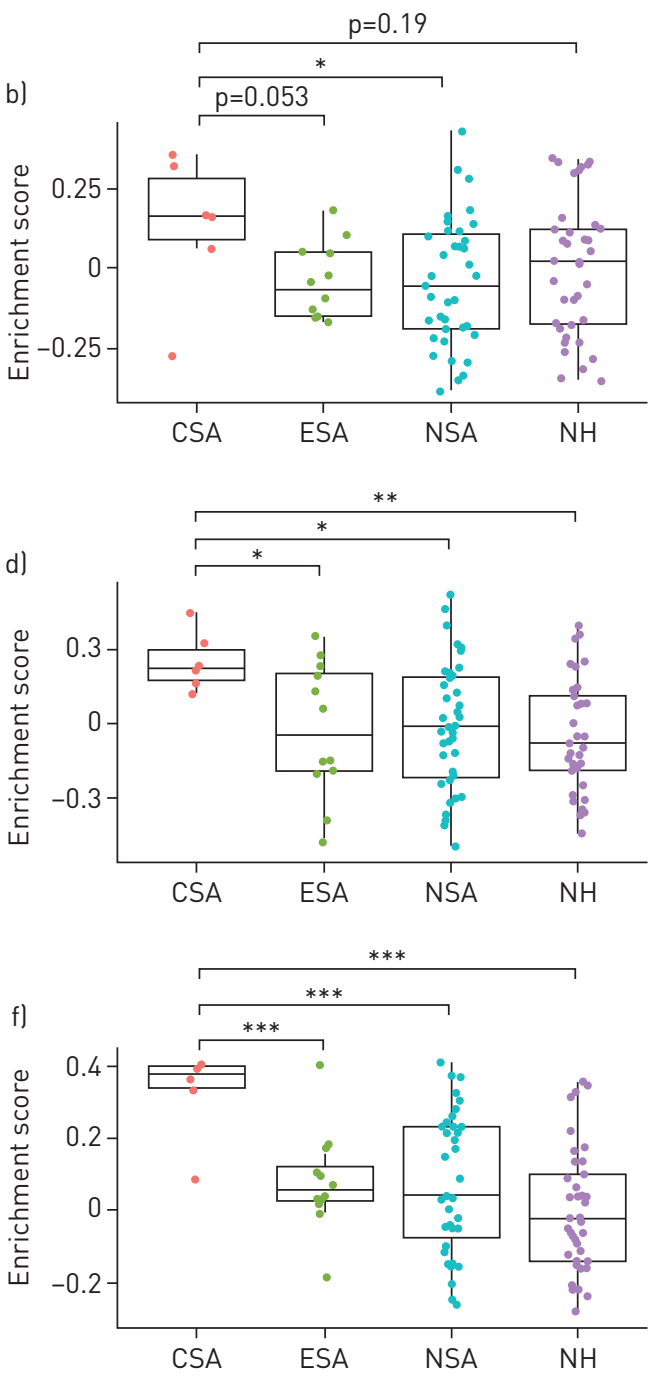

FIGURE 5 Gene set variation analysis of selected stress-related pathways in bronchial brushings according to smoking status. Box-and-whisker plots showing pathway enrichment of al xenobiotic metabolism by CYP450, b) glutathione metabolism, c) response to oxidative stress, d) endoplasmic reticulum stress, e] unfolded protein response, f) lysosome and g) glycolysis and gluconeogenesis in bronchial brushings of severe asthma current smokers (CSA), severe asthma ex-smokers (ESA), severe asthma nonsmokers (NSA) and healthy nonsmokers $(\mathrm{NH}) .^{*}: \mathrm{p}<0.05 ;{ }^{* *}: \mathrm{p}<0.01 ;^{* * *}: \mathrm{p}<0.001$.

In the biopsies, 16 DEGs (fold change $\geqslant 2.0$ ) were detected and these included follicular dendritic cell secreted protein (FDCSP), periostin (POSTN), PPBP, immunoglobulin $\lambda$ constant 1 (IGLC1) and immunoglobulin $\lambda$ variable cluster (IGLV). FDCSP and PPBP were upregulated in ESA subjects, while POSTN, IGLC1 and IGLV were downregulated. Overall, the data suggest that neutrophilic innate immunity is more characteristic of ESA than IL-4/13 signalling and humoral immunity. 

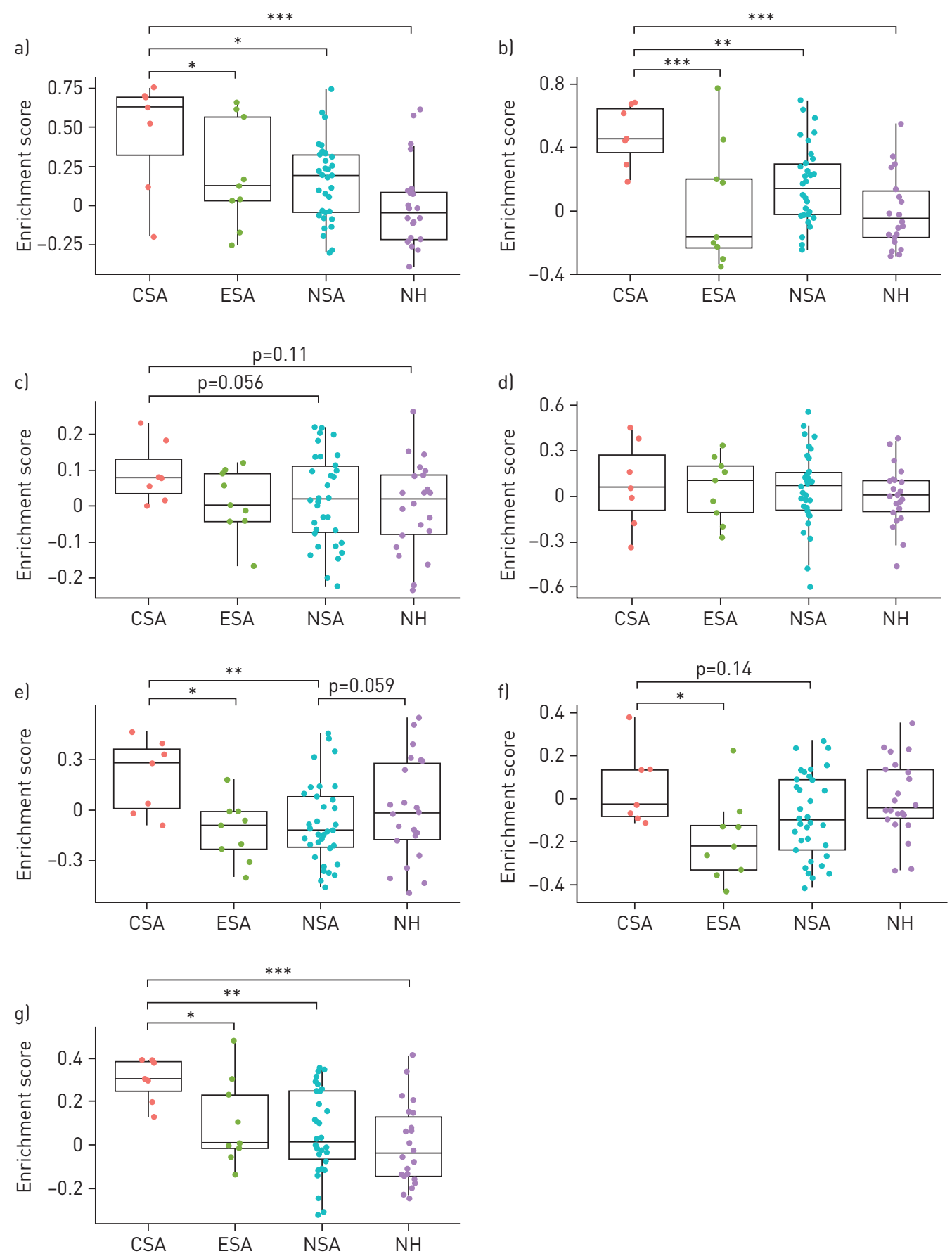

FIGURE 6 Gene set variation analysis of selected stress-related pathways in bronchial biopsies according to smoking status. Box-and-whisker plots showing pathway enrichment of al xenobiotic metabolism by CYP450, b) glutathione metabolism, c) response to oxidative stress, d) endoplasmic reticulum stress, e) unfolded protein response, fl lysosome and gl glycolysis and gluconeogenesis in bronchial biopsies of severe asthma current smokers (CSA), severe asthma ex-smokers (ESA), severe asthma nonsmokers (NSA) and healthy nonsmokers $(\mathrm{NH}) .^{*}: \mathrm{p}<0.05{ }^{* *}: \mathrm{p}<0.01 ;^{* * *}: \mathrm{p}<0.001$.

\section{Protein interaction analysis using combined DEGs from airway samples}

Protein interaction analysis in STRING using combined DEGs between CSA and NSA showed direct interactions of oxidation-reduction and the pentose phosphate pathway network with the innate immune response via protein production and modification in endoplasmic reticulum (figure 7). In addition, proteins that play a role in lysosome, mucus production, Golgi homeostasis and tissue structure were seen in the network. 


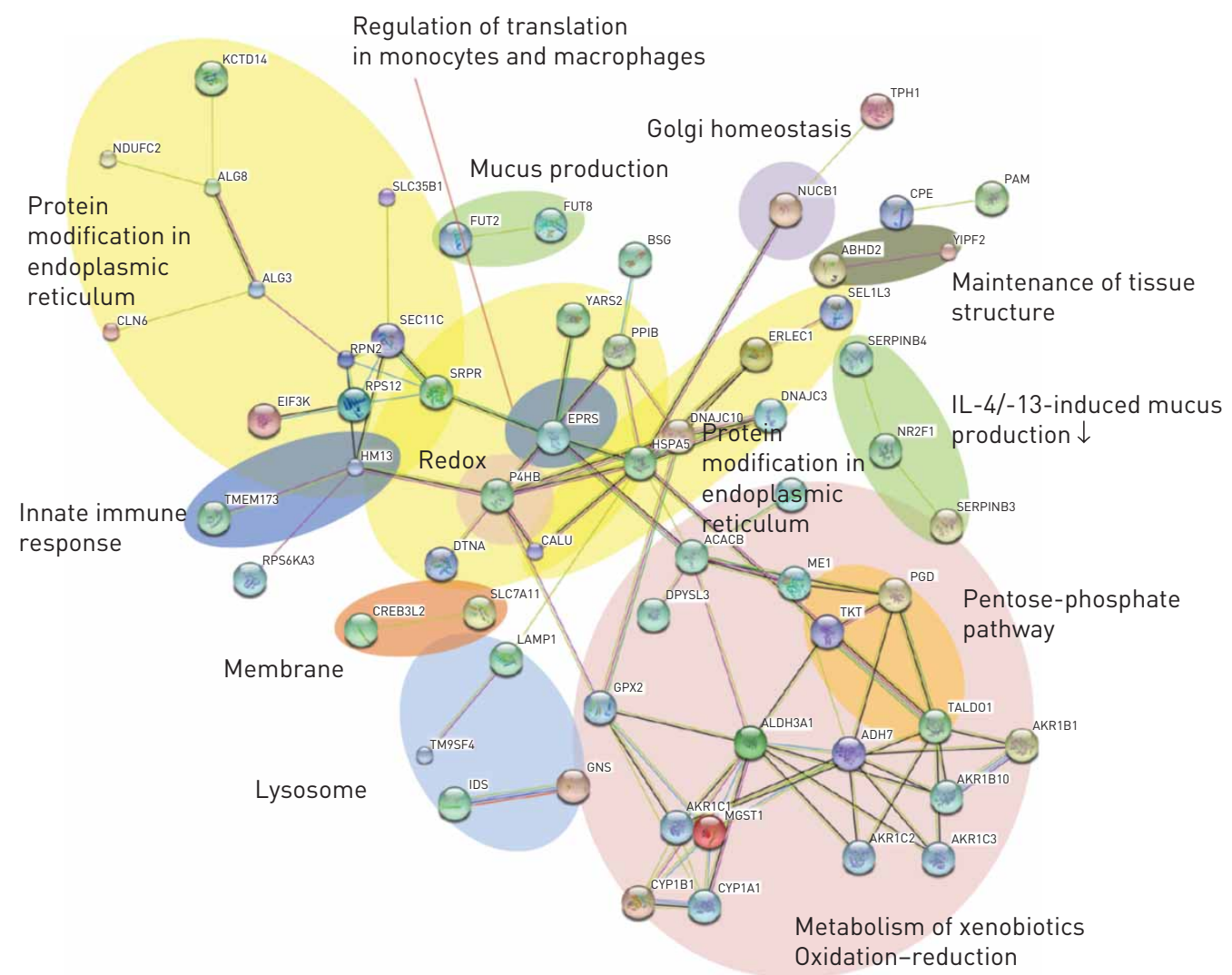

FIGURE 7 Protein interaction analysis in STRING (version 10.0; STRING Consortium 2016, www.string-db.org) using combined differentially expressed genes (DEGs). Combined DEGs in limma from bronchial brushings, biopsies and sputa were used for protein interaction analysis by STRING. The large pink-coloured area is filled with proteins related to xenobiotic metabolism and oxidation-reduction which contains the pentose-phosphate pathway. These proteins function with those in charge of redox and connect with protein production or modification. Some proteins are associated with innate immunity. The other proteins function as lysosomal, membranous, mucus productive, Golgi homeostatic or structural proteins. Overall, this reveals the relationship between oxidative stress, endoplasmic reticulum stress, metabolism of xenobiotics and innate immunity.

\section{Discussion}

We describe the differences in protein and gene expression between severe asthma patients who actively smoke, ex-smokers with a significant history of cigarette smoking and those who do not smoke. There was a difference in the sputum proteome between NSA and CSA (CSF2, AGR2 and CXCL8) and between NSA and ESA (AZU1, ELANE, CFP and CXCL8) subjects, with CXCL8 not discriminating between ESA and CSA. Distinct pathways were activated in CSA and NSA sputum, while the sputum protein data also suggested that ESA was associated with airway epithelial cell damage. In addition, gene expression profiles between bronchial epithelial cells from CSA and NSA were significantly different as determined by pathway analysis, GSVA and protein-protein interaction analysis. There were no significant DEGs (FDR $<0.05$ ) between ESA and NSA. Hierarchical clustering indicated that although CSA and NSA were clearly distinct, NSA and ESA did not cluster separately. Airway epithelial cells in CSA patients show an enrichment of oxidative and endoplasmic reticulum stress and innate immune pathways compared to ESA or NSA patients and there were no significant differences between ESA and NSA for these pathways. Using a less stringent analysis, ESA subjects showed upregulated expression of neutrophil chemotactic genes and downregulated expression of genes related to mast cells, humoral immunity and epithelial protection compared to NSA. Overall, proteomics and transcriptomics were able to differentiate CSA from NSA, but ESA and NSA could only be discriminated using sputum proteomics, as airway transcriptomics clustered ESA and CSA together.

The role of the increased sputum expression of CSF2 is unknown. CSF2 is secreted by macrophages, epithelial cells and T-cells in response to inflammatory and noxious stimuli and its expression is enhanced in asthmatic airway epithelial cells in situ and after culture [26]. CSF2 transgenic mice have an enhanced Th2 response to ovalbumin sensitisation and anti-CSF2 antibodies block the allergic response in mouse 
models of asthma [27]. In addition, CSF2 is involved in the lung innate immune response to noxious agents such as lipopolysaccharide and cigarette smoke [28]. Acute exposure to cigarette smoke in mice leads to enhanced CSF2 expression, and neutralisation using an intranasal anti-CSF2 antibody reduced bronchoalveolar lavage fluid macrophages and neutrophils and inflammatory analytes [29], which indicates that the CSF2 pathway can mediate smoke-induced inflammation. Future experiments in models of severe asthma linked to smoking or in selected patients may determine whether the elevated CSF2 expression seen here is causal or a marker of other driver mechanisms.

Our data provide evidence for enhanced oxidative and endoplasmic reticulum stress in airway epithelial cells of CSA patients. We postulate that the increased activation of the xenobiotic response and oxidative and endoplasmic reticulum stress pathways influences innate immunity in these subjects. There is increased oxidative stress in asthma and COPD patients as well as in healthy smokers [30]. Cigarette smoke not only contains high concentrations of reactive oxygen species (ROS) [30], but also activates alveolar macrophages and neutrophils, which also release ROS, leading to an increased inflammatory response in a feed-forward process $[30,31]$. In both asthma and COPD, activated inflammatory cells including neutrophils, macrophages and eosinophils produce ROS and further generate inflammation and cause injury to the airway epithelium [30]. Moreover, impaired upregulation and production of protective antioxidant was reported in smokers, asthma and COPD patients. This oxidant-antioxidant imbalance resulting in oxidative stress is associated with airway hyperresponsiveness and decreased lung function and asthma severity [30, 32].

CSA represented the escalated response to oxidative stress derived from cigarette smoking as CSA bronchial brushings and biopsies alone were enriched for the active smoking-related gene set, whereas both CSA and ESA samples showed a similar enrichment of the pack-year signature. Increased antioxidant gene expression and increased enrichment of the gene set showing response to oxidative stress were observed in bronchial brushings, which may suggest that cigarette smoking stimulates airway epithelial cells to respond to oxidative stress in severe asthma. In addition, we showed that endoplasmic reticulum stress might have a key role in CSA phenotype. Endoplasmic reticulum stress is associated with neutrophilic asthma through nuclear factor- $\mathrm{\kappa B}$ activation and pro-inflammatory cytokine production [33]. Cigarette smoking itself may induce endoplasmic reticulum stress [34] and the activation seen here in severe asthma might relate to active cigarette smoke exposure. However, CSF2 and AGR2 have not been shown to be differentially expressed in the previously published proteomic analysis of sputum from healthy current smokers compared to never-smokers [35]. Moreover, endoplasmic stress and lysosome gene sets that we found to be differentially expressed in these two groups of severe asthma were not differentially expressed in healthy current smokers compared to nonsmokers (online supplementary tables S9 and S10) [21]. These results suggest that DEGs between CSA and NSA were not derived from the influence of cigarette smoking itself.

We found decreased production of protective agents in ESA airways. Cigarette smoke injures the airway epithelium in several ways, including decreased protective protein expression [36], disruption of tight junctions [37] and through innate immune and inflammatory response [31]. Cigarette smoke-activated alveolar macrophages produce pro-inflammatory molecules, ROS, tissue proteases and chemokines for recruitment and survival of neutrophils in the lung tissue [31] and activated neutrophils secrete proteases and breakdown collagen into fragments, which can activate neutrophils in a positive-feedback manner [38]. We showed decreased expression of MUC2 and CST1 in ESA, which both play a protective role for airway epithelium $[39,40]$. Conversely, the expression of MMP12, CXCL8 and PPBP, which can enhance lung damage, were upregulated in ESA.

Sputum microbiota, which is associated with neutrophilic airway inflammation in adult severe asthma has been reported to be different from that of healthy controls or nonsevere asthmatics [41, 42]. Our results imply that a reduction in airway protective agents might change the airway microbiome, affecting neutrophilic airway inflammation, especially in ESA; conversely, the heightened mucin production might have had a beneficial effect in keeping the airway epithelium free from bacterial colonisation in CSA.

There are important limitations in our study. First, the numbers of smoking and ex-smokers in our groups were relatively small, particularly when analysing data from sputum and biopsy and brushing samples, and the results should be considered as exploratory and will need confirmation in a larger cohort. Second, the lack of a control group of age-matched nonasthmatic active smokers does not allow us to determine the exact contribution of cigarette smoking to the changes observed. Third, we did not observe differences in blood or sputum neutrophil counts, although neutrophil chemoattractants were more upregulated in airways of ESA and CSA patients compared to controls.

In conclusion, we found that current smokers with severe asthma were characterised by increased sputum CFS2 and AGR2 protein expression, indicating enhanced macrophage recruitment and mucus production 
in addition to airway tissue genes associated with increased xenobiotic metabolism and responses to oxidative stress and endoplasmic reticulum stress. In contrast, ex-smokers with severe asthma were characterised by pathways involved in the recruitment and activity of neutrophils and with decreased airway protective factors. Airway gene expression analysis showed little difference between severe asthmatics who were ex-smokers and those who were never-smokers.

Acknowledgements: The U-BIOPRED consortium wishes to acknowledge the help and expertise of the following individuals and groups without whom the study would not have been possible: I.M. Adcock, National Heart and Lung Institute, Imperial College, London, UK; H. Ahmed, European Institute for Systems Biology and Medicine, CNRS-ENS-UCBL-INSERM, Lyon, France; C. Auffray, European Institute for Systems Biology and Medicine, CNRS-ENS-UCBL-INSERM, Lyon, France; P. Bakke, Dept of Clinical Science, University of Bergen, Bergen, Norway; A.T. Banssal, Acclarogen Ltd, St John's Innovation Centre, Cambridge, UK; F. Baribaud, Janssen R\&D, USA; S. Bates, Respiratory Therapeutic Unit, GSK, London, UK; E.H. Bel, Academic Medical Centre, University of Amsterdam, Amsterdam, The Netherlands; J. Bigler, previously Amgen Inc.; H. Bisgaard, COPSAC, Copenhagen Prospective Studies on Asthma in Childhood, Herlev and Gentofte Hospital, University of Copenhagen, Copenhagen, Denmark; M.J. Boedigheimer, Amgen Inc., Thousand Oaks, USA; K. Bønnelykke, COPSAC, Copenhagen Prospective Studies on Asthma in Childhood, Herlev and Gentofte Hospital, University of Copenhagen, Copenhagen, Denmark; J. Brandsma, University of Southampton, Southampton, UK; P. Brinkman, Academic Medical Centre, University of Amsterdam, Amsterdam, The Netherlands; E. Bucchioni, Chiesi Pharmaceuticals SPA, Parma, Italy; D. Burg, Centre for Proteomic Research, Institute for Life Sciences, University of Southampton, Southampton, UK; A. Bush, National Heart and Lung Institute, Imperial College, London, UK; Royal Brompton and Harefield NHS trust, UK; M. Caruso, Dept Clinical and Experimental Medicine, University of Catania, Catania, Italy; A. Chaiboonchoe, European Institute for Systems Biology and Medicine, CNRS-ENS-UCBL-INSERM, Lyon, France; P. Chanez, Assistance publique des Hôpitaux de Marseille - Clinique des bronches, allergies et sommeil, Aix Marseille Université, Marseille, France; K.F. Chung, National Heart and Lung Institute, Imperial College, London, UK; C.H. Compton, Respiratory Therapeutic Unit, GSK, London, UK; J. Corfield, Areteva R\&D, Nottingham, UK; A. D’Amico, University of Rome 'Tor Vergata', Rome Italy; S.E. Dahlen, Centre for Allergy Research, Karolinska Institutet, Stockholm, Sweden; B. De Meulder, European Institute for Systems Biology and Medicine, CNRS-ENS-UCBL-INSERM, Lyon, France; R. Djukanovic, NIHR Southampton Respiratory Biomedical Research Unit and Clinical and Experimental Sciences, Southampton, UK; V.J. Erpenbeck, Translational Medicine, Respiratory Profiling, Novartis Institutes for Biomedical Research, Basel, Switzerland; D. Erzen, Boehringer Ingelheim Pharma GmbH \& Co. KG, Biberach, Germany; K. Fichtner, Boehringer Ingelheim Pharma GmbH \& Co. KG, Biberach, Germany; N. Fitch, BioSci Consulting, Maasmechelen, Belgium; L.J. Fleming, National Heart and Lung Institute, Imperial College, London, UK; Royal Brompton and Harefield NHS trust, UK; E. Formaggio, previously CROMSOURCE, Verona, Italy; S.J. Fowler, Centre for Respiratory Medicine and Allergy, Institute of Inflammation and Repair, University of Manchester and University Hospital of South Manchester, Manchester Academic Health Sciences Centre, Manchester, UK; U. Frey, University Children's Hospital, Basel, Switzerland; M. Gahlemann, Boehringer Ingelheim (Schweiz) GmbH, Basel, Switzerland; T. Geiser, Dept of Respiratory Medicine, University Hospital Bern, Switzerland; Y. Guo, Data Science Institute, Imperial College, London, UK; S. Hashimoto, Academic Medical Centre, University of Amsterdam, Amsterdam, The Netherlands; J. Haughney, International Primary Care Respiratory Group, Aberdeen, UK; G. Hedlin, Dept Women's and Children's Health \& Centre for Allergy Research, Karolinska Institutet, Stockholm, Sweden; P.W. Hekking, Academic Medical Centre, University of Amsterdam, Amsterdam, The Netherlands; T. Higenbottam, Allergy Therapeutics, West Sussex, UK; J.M. Hohlfeld, Fraunhofer Institute for Toxicology and Experimental Medicine, Hannover, Germany; C. Holweg, Respiratory and Allergy Diseases, Genentech, San Francisco, USA; I. Horváth, Semmelweis University, Budapest, Hungary; P. Howarth, NIHR Southampton Respiratory Biomedical Research Unit, Clinical and Experimental Sciences and Human Development and Health, Southampton, UK; A.J. James, Centre for Allergy Research, Karolinska Institutet, Stockholm, Sweden; R. Knowles, Arachos Pharma, Stevenge, UK; A. J. Knox, Respiratory Research Unit, University of Nottingham, Nottingham, UK; N. Krug, Fraunhofer Institute for Toxicology and Experimental Medicine, Hannover, Germany; D. Lefaudeux, European Institute for Systems Biology and Medicine, CNRS-ENS-UCBL-INSERM, Lyon, France; M.J. Loza, Janssen R\&D, USA; R. Lutter, Academic Medical Centre, University of Amsterdam, Amsterdam, The Netherlands; A. Manta, Roche Diagnostics GmbH, Mannheim, Germany; S. Masefield, European Lung Foundation, Sheffield, UK; J.G. Matthews, Respiratory and Allergy Diseases, Genentech, San Francisco, USA; A. Mazein, European Institute for Systems Biology and Medicine, CNRS-ENS-UCBL-INSERM, Lyon, France; A. Meiser, Data Science Institute, Imperial College, London, UK; R.J.M. Middelveld, Centre for Allergy Research, Karolinska Institutet, Stockholm, Sweden; M. Miralpeix, Almirall, Barcelona, Spain; P. Montuschi, Università Cattolica del Sacro Cuore, Milan, Italy; N. Mores, Università Cattolica del Sacro Cuore, Milan, Italy; C.S. Murray, Centre for Respiratory Medicine and Allergy, Institute of Inflammation and Repair, University of Manchester and University Hospital of South Manchester, Manchester Academic Health Sciences Centre, Manchester, UK; J. Musial, Dept of Medicine, Jagiellonian University Medical College, Krakow, Poland; D. Myles, Respiratory Therapeutic Unit, GSK, London, UK; L. Pahus, Assistance publique des Hôpitaux de Marseille, Clinique des bronches, allergies et sommeil, Espace Éthique Méditerranéen, Aix-Marseille Université, Marseille, France; I. Pandis, Data Science Institute, Imperial College, London, UK; S. Pavlidis, National Heart and Lung Institute, Imperial College, London, UK; P. Powell, European Lung Foundation, Sheffield, UK; G. Praticò, CROMSOURCE, Verona, Italy; M. Puig Valls, CROMSOURCE, Barcelona, Spain; N. Rao, Janssen R\&D, USA; J. Riley, Respiratory Therapeutic Unit, GSK, London, UK; A. Roberts, Asthma UK, London, UK; G. Roberts, NIHR Southampton Respiratory Biomedical Research Unit, Clinical and Experimental Sciences and Human Development and Health, Southampton, UK; A. Rowe, Janssen R\&D, UK; T. Sandström, Dept of Public Health and Clinical Medicine, Umeå University, Umeå, Sweden; W. Seibold, Boehringer Ingelheim Pharma GmbH, Biberach, Germany; A. Selby, NIHR Southampton Respiratory Biomedical Research Unit, Clinical and Experimental Sciences and Human Development and Health, Southampton, UK; D.E. Shaw, Respiratory Research Unit, University of Nottingham, UK; R. Sigmund, Boehringer Ingelheim Pharma GmbH \& Co. KG, Biberach, Germany; F. Singer, University Children's Hospital, Zurich, Switzerland; P.J. Skipp, Centre for Proteomic Research, Institute for Life Sciences, University of Southampton, Southampton, UK; A.R. Sousa, Respiratory Therapeutic Unit, GSK, London, UK; P.J. Sterk, Academic Medical Centre, University of Amsterdam, Amsterdam, The Netherlands; K. Sun, Data Science Institute, Imperial College, London, UK; B. Thornton, MSD, USA; W.M. van Aalderen, Academic Medical Centre, University of Amsterdam, Amsterdam, The Netherlands; M. van Geest, AstraZeneca, Mölndal, Sweden; J. Vestbo, Centre for Respiratory Medicine and Allergy, Institute of Inflammation and Repair, University of Manchester and University Hospital of South Manchester, Manchester Academic Health Sciences Centre, Manchester, UK; N.H. Vissing, COPSAC, Copenhagen Prospective Studies on 
Asthma in Childhood, Herlev and Gentofte Hospital, University of Copenhagen, Copenhagen, Denmark; A.H. Wagener, Academic Medical Center Amsterdam, Amsterdam, The Netherlands; S.S. Wagers, BioSci Consulting, Maasmechelen, Belgium; Z. Weiszhart, Semmelweis University, Budapest, Hungary; C.E. Wheelock, Centre for Allergy Research, Karolinska Institutet, Stockholm, Sweden; S.J. Wilson, Histochemistry Research Unit, Faculty of Medicine, University of Southampton, Southampton, UK. Additional contributors: Antonios Aliprantis, Merck Research Laboratories, Boston, USA; David Allen, North West Severe Asthma Network, Pennine Acute Hospital NHS Trust, UK; Kjell Alving, Dept Women's \& Children's Health, Uppsala University, Uppsala, Sweden; P. Badorrek, Fraunhofer ITEM, Hannover, Germany; David Balgoma, Centre for Allergy Research, Karolinska Institutet, Stockholm, Sweden; S. Ballereau, European institute for Systems Biology and Medicine, University of Lyon, France; Clair Barber, NIHR Southampton Respiratory Biomedical Research Unit and Clinical and Experimental Sciences, Southampton, UK; Manohara Kanangana Batuwitage, Data Science Institute, Imperial College, London, UK; An Bautmans, MSD, Brussels, Belgium; A. Bedding, Roche Diagnostics GmbH, Mannheim, Germany; A.F. Behndig, Umeå University, Umea, Sweden; Jorge Beleta, Almirall S.A., Barcelona, Spain; A. Berglind, MSD, Brussels, Belgium; A. Berton, AstraZeneca, Mölndal, Sweden; Grazyna Bochenek, II Dept of Internal Medicine, Jagiellonian University Medical College, Krakow, Poland; Armin Braun, Fraunhofer Institute for Toxicology and Experimental Medicine, Hannover, Germany; D. Campagna, Dept of Clinical and Experimental Medicine, University of Catania, Catania, Italy; Leon Carayannopoulos, previously at MSD, USA; C. Casaulta, University Children's Hospital of Bern, Switzerland; Romanas Chaleckis, Centre of Allergy Research, Karolinska Institutet, Stockholm, Sweden; B. Dahlén, Karolinska University Hospital \& Centre for Allergy Research, Karolinska Institutet, Stockholm, Sweden; Timothy Davison, Janssen R\&D, USA; Jorge De Alba, Almirall S.A., Barcelona, Spain; Inge De Lepeleire, MSD, Brussels, Belgium; Tamara Dekker, Academic Medical Centre, University of Amsterdam, Amsterdam, The Netherlands; Ingrid Delin, Centre for Allergy Research, Karolinska Institutet, Stockholm, Sweden; P. Dennison, NIHR Southampton Respiratory Biomedical Research Unit, Clinical and Experimental Sciences, NIHR-Wellcome Trust Clinical Research Facility, Faculty of Medicine, University of Southampton, Southampton, UK; Annemiek Dijkhuis, Academic Medical Centre, University of Amsterdam, Amsterdam, The Netherlands; Paul Dodson, AstraZeneca, Mölndal, Sweden; Aleksandra Draper, BioSci Consulting, Maasmechelen, Belgium; K. Dyson, CROMSOURCE, Stirling, UK; Jessica Edwards, Asthma UK, London, UK; L. El Hadjam, European Institute for Systems Biology and Medicine, University of Lyon, France; Rosalia Emma, Dept of Clinical and Experimental Medicine, University of Catania, Catania, Italy; Magnus Ericsson, Karolinska University Hospital, Stockholm, Sweden; C. Faulenbach, Fraunhofer ITEM, Hannover, Germany; Breda Flood, European Federation of Allergy and Airways Diseases Patient's Associations, Brussels, Belgium; G. Galffy, Semmelweis University, Budapest, Hungary; Hector Gallart, Centre for Allergy Research, Karolinska Institutet, Stockholm, Sweden; D. Garissi, Global Head Clinical Research Division, CROMSOURCE, Italy; J. Gent, Royal Brompton and Harefield NHS Foundation Trust, London, UK; M. Gerhardsson de Verdier, AstraZeneca; Mölndal, Sweden; D. Gibeon, National Heart and Lung Institute, Imperial College, London, UK; Cristina Gomez, Centre for Allergy Research, Karolinska Institutet, Stockholm, Sweden; Kerry Gove, NIHR Southampton Respiratory Biomedical Research Unit and Clinical and Experimental Sciences, Southampton, UK; Neil Gozzard, UCB, Slough, UK; E. Guillmant-Farry, Royal Brompton Hospital, London, UK; E. Henriksson, Karolinska University Hospital \& Karolinska Institutet, Stockholm, Sweden; Lorraine Hewitt, NIHR Southampton Respiratory Biomedical Research Unit, Southampton, UK; U. Hoda, Imperial College, London, UK; Richard Hu, Amgen Inc. Thousand Oaks, USA; Sile Hu, National Heart and Lung Institute, Imperial College, London, UK; X. Hu, Amgen Inc., Thousand Oaks, USA; E. Jeyasingham, UK Clinical Operations, GSK, Stockley Park, UK; K. Johnson, Centre for Respiratory Medicine and Allergy, Institute of Inflammation and repair, University Hospital of South Manchester, NHS Foundation Trust, Manchester, UK; N. Jullian, European Institute for Systems Biology and Medicine, University of Lyon, France; Juliette Kamphuis, Longfonds, Amersfoort, The Netherlands; Erika J. Kennington, Asthma UK, London, UK; Dyson Kerry, CromSource, Stirling, UK; G. Kerry, Centre for Respiratory Medicine and Allergy, Institute of Inflammation and Repair, University Hospital of South Manchester, NHS Foundation Trust, Manchester, UK; M. Klüglich, Boehringer Ingelheim Pharma GmbH \& Co. KG, Biberach, Germany; Hugo Knobel, Philips Research Laboratories, Eindhoven, The Netherlands; Johan Kolmert, Centre for Allergy Research, Karolinska Institutet, Stockholm, Sweden; J.R. Konradsen, Dept Women's and Children's Health \& Centre for Allergy Research, Karolinska Institutet, Stockholm, Sweden; Maxim Kots, Chiesi Pharmaceuticals, SPA, Parma, Italy; Kosmas Kretsos, UCB, Slough, UK; L. Krueger, University Children's Hospital Bern, Switzerland; Scott Kuo, National Heart and Lung Institute, Imperial College, London, UK; Maciej Kupczyk, Centre for Allergy Research, Karolinska Institutet, Stockholm, Sweden; Bart Lambrecht, University of Gent, Gent, Belgium; A-S. Lantz, Karolinska University Hospital \& Centre for Allergy Research, Karolinska Institutet, Stockholm, Sweden; Christopher Larminie, GSK, London, UK; L.X. Larsson, AstraZeneca, Mölndal, Sweden; P. Latzin, University Children's Hospital of Bern, Bern, Switzerland; N. Lazarinis, Karolinska University Hospital \& Karolinska Institutet, Stockholm, Sweden; N. Lemonnier, European Institute for Systems Biology and Medicine, CNRS-ENS-UCBL-INSERM, Lyon, France; Saeeda Lone-Latif, Academic Medical Centre, University of Amsterdam, Amsterdam, The Netherlands; L.A. Lowe, Centre for Respiratory Medicine and Allergy, Institute of Inflammation and Repair, University Hospital of South Manchester, NHS Foundation Trust, Manchester, UK; Alexander Manta, Roche Diagnostics GmbH, Mannheim, Germany; Lisa Marouzet, NIHR Southampton Respiratory Biomedical Research Unit, Southampton, UK; Jane Martin, NIHR Southampton Respiratory Biomedical Research Unit, Southampton, UK; Caroline Mathon, Centre of Allergy Research, Karolinska Institutet, Stockholm, Sweden; L. McEvoy, University Hospital, Dept of Pulmonary Medicine, Bern, Switzerland; Sally Meah, National Heart and Lung Institute, Imperial College, London, UK; A. Menzies-Gow, Royal Brompton and Harefield NHS Foundation Trust, London, UK; Leanne Metcalf, previously at Asthma UK, London, UK; Maria Mikus, Science for Life Laboratory \& The Royal Institute of Technology, Stockholm, Sweden; Philip Monk, Synairgen Research Ltd, Southampton, UK; Shama Naz, Centre for Allergy Research, Karolinska Institutet, Stockholm, Sweden; K. Nething, Boehringer Ingelheim Pharma GmbH \& Co. KG, Biberach, Germany; Ben Nicholas, University of Southampton, Southampton, UK; U. Nihlén, previously at AstraZeneca, Mölndal, Sweden; Peter Nilsson, Science for Life Laboratory \& The Royal Institute of Technology, Stockholm, Sweden; R. Niven, North West Severe Asthma Network, University Hospital South Manchester, UK; B. Nordlund, Dept Women's and Children's Health \& Centre for Allergy Research, Karolinska Institutet, Stockholm, Sweden; S. Nsubuga, Royal Brompton Hospital, London, UK; Jörgen Östling, AstraZeneca, Mölndal, Sweden; Antonio Pacino, Lega Italiano Anti Fumo, Catania, Italy; Susanna Palkonen, European Federation of Allergy and Airways Diseases Patient's Associations, Brussels, Belgium; J. Pellet, European Institute for Systems Biology and Medicine, CNRS-ENS-UCBL-INSERM, Lyon, France; Giorgio Pennazza, University of Rome 'Tor Vergata', Rome Italy; Anne Petrén, Centre for Allergy Research, Karolinska Institutet, Stockholm, Sweden; Sandy Pink, NIHR Southampton Respiratory Biomedical Research Unit, Southampton, UK; C. Pison, European Institute for Systems Biology and Medicine, CNRS-ENS-UCBL-INSERM, Lyon, France; Anthony Postle, University of Southampton, UK; Malayka Rahman-Amin, previously at Asthma UK, London, UK; Lara Ravanetti, Academic Medical 
Centre, University of Amsterdam, Amsterdam, The Netherlands; Emma Ray, NIHR Southampton Respiratory Biomedical Research Unit, Southampton, UK; Stacey Reinke, Centre for Allergy Research, Karolinska Institutet, Stockholm, Sweden; Leanne Reynolds, previously at Asthma UK, London, UK; K. Riemann, Boehringer Ingelheim Pharma GmbH \& Co. KG, Biberach, Germany; Martine Robberechts, MSD, Brussels, Belgium; J.P. Rocha, Royal Brompton and Harefield NHS Foundation Trust, UK; C. Rossios, National Heart and Lung Institute, Imperial College, London, UK; Kirsty Russell, National Heart and Lung Institute, Imperial College, London, UK; Michael Rutgers, Longfonds, Amersfoort, The Netherlands; G. Santini, Università Cattolica del Sacro Cuore, Milan, Italy; Marco Santoninco, University of Rome 'Tor Vergata', Rome Italy; M. Saqi, European Institute for Systems Biology and Medicine, CNRS-ENS-UCBL-INSERM, Lyon, France; Corinna Schoelch, Boehringer Ingelheim Pharma GmbH \& Co. KG, Biberach, Germany; James P.R. Schofield, Centre for Proteomic Research, Institute for Life Sciences, University of Southampton, Southampton, UK; S. Scott, North West Severe Asthma Network, Countess of Chester Hospital, UK; N. Sehgal, North West Severe Asthma Network, Pennine Acute Hospital NHS Trust; Marcus Sjödin, Centre for Allergy Research, Karolinska Institutet, Stockholm, Sweden; Barbara Smids, Academic Medical Centre, University of Amsterdam, Amsterdam, The Netherlands; Caroline Smith, NIHR Southampton Respiratory Biomedical Research Unit, Southampton, UK; Jessica Smith, Asthma UK, London, UK; Katherine M. Smith, University of Nottingham, UK; P. Söderman, Dept Women's and Children's Health, Karolinska Institutet, Stockholm, Sweden; A. Sogbessan, Royal Brompton and Harefield NHS Foundation Trust, London, UK; F. Spycher, University Hospital Dept of Pulmonary Medicine, Bern, Switzerland; Doroteya Staykova, University of Southampton, Southampton, UK; S. Stephan, Centre for Respiratory Medicine and Allergy, Institute of Inflammation and Repair, University Hospital of South Manchester, NHS Foundation Trust, Manchester, UK; J. Stokholm, University of Copenhagen and Danish Pediatric Asthma Centre Denmark; K. Strandberg, Karolinska University Hospital \& Karolinska Institutet, Stockholm, Sweden; M. Sunther, Centre for Respiratory Medicine and Allergy, Institute of Inflammation and Repair, University Hospital of South Manchester, NHS Foundation Trust, Manchester, UK; M. Szentkereszty, Semmelweis University, Budapest, Hungary; L. Tamasi, Semmelweis University, Budapest, Hungary; K. Tariq, NIHR Southampton Respiratory Biomedical Research Unit, Clinical and Experimental Sciences, NIHR-Wellcome Trust Clinical Research Facility, Faculty of Medicine, University of Southampton, Southampton, UK; John-Olof Thörngren, Karolinska University Hospital, Stockholm, Sweden; Jonathan Thorsen, COPSAC, Copenhagen Prospective Studies on Asthma in Childhood, Herlev and Gentofte Hospital, University of Copenhagen, Copenhagen, Denmark; S. Valente, Università Cattolica del Sacro Cuore, Milan, Italy; Marianne van de Pol, Academic Medical Centre, University of Amsterdam, Amsterdam, The Netherlands; C.M. van Drunen, Academic Medical Centre, University of Amsterdam, Amsterdam, The Netherlands; Jonathan Van Eyll, UCB, Slough, UK; Jenny Versnel, previously at Asthma UK, London, UK; Anton Vink, Philips Research Laboratories, Eindhoven, The Netherlands; C. von Garnier, University Hospital Bern, Switzerland; A. Vyas, North West Severe Asthma Network, Lancashire Teaching Hospitals NHS Trust, UK; Frans Wald, Boehringer Ingelheim Pharma GmbH \& Co. KG, Biberach, Germany; Samantha Walker, Asthma UK, London, UK; Jonathan Ward, Histochemistry Research Unit, Faculty of Medicine, University of Southampton, Southampton, UK; Kristiane Wetzel, Boehringer Ingelheim Pharma GmbH, Biberach, Germany; Coen Wiegman, National Heart and Lung Institute, Imperial College, London, UK; Siân Williams, International Primary Care Respiratory Group, Aberdeen, UK; Xian Yang, Data Science Institute, Imperial College, London, UK; Elizabeth Yeyasingham, UK Clinical Operations, GSK, Stockley Park, UK; W. Yu, Amgen Inc., Thousand Oaks, USA; W. Zetterquist, Dept Women's and Children's Health \& Centre for Allergy Research, Karolinska Institutet, Stockholm, Sweden; Z. Zolkipli, NIHR Southampton Respiratory Biomedical Research Unit, Clinical and Experimental Sciences and Human Development and Health, Southampton, UK; A.H. Zwinderman, Academic Medical Centre, University of Amsterdam, The Netherlands. Partner organisations: Novartis Pharma AG; University of Southampton, Southampton, UK; Academic Medical Centre, University of Amsterdam, Amsterdam, The Netherlands; Imperial College London, London, UK; University of Catania, Catania, Italy; University of Rome 'Tor Vergata', Rome, Italy; Hvidore Hospital, Hvidore, Denmark; Jagiellonian Univ. Medi. College, Krakow, Poland; University Hospital, Inselspital, Bern, Switzerland; Semmelweis University, Budapest, Hungary; University of Manchester, Manchester, UK; Université d'Aix-Marseille, Marseille, France; Fraunhofer Institute, Hannover, Germany; University Hospital, Umea, Sweden; Ghent University, Ghent, Belgium; Ctr. Nat. Recherche Scientifique, Lyon, France; Università Cattolica del Sacro Cuore, Rome, Italy; University Hospital, Copenhagen, Denmark; Karolinska Institutet, Stockholm, Sweden; Nottingham University Hospital, Nottingham, UK; University of Bergen, Bergen, Norway; Netherlands Asthma Foundation, Leusden, The Netherlands; European Lung Foundation, Sheffield, UK; Asthma UK, London, UK; European. Fed. of Allergy and Airways Diseases Patients' Associations, Brussels, Belgium; Lega Italiano Anti Fumo, Catania, Italy; International Primary Care Respiratory Group, Aberdeen, UK; Philips Research Laboratories, Eindhoven, The Netherlands; Synairgen Research Ltd, Southampton, UK; Aerocrine AB, Stockholm, Sweden; BioSci Consulting, Maasmechelen, Belgium; Almirall; AstraZeneca; Boehringer Ingelheim; Chiesi; GlaxoSmithKline; Roche; UCB; Janssen Biologics BV; Amgen NV; Merck Sharp \& Dome Corp. Members of the ethics board: Jan-Bas Prins, biomedical research, LUMC, The Netherlands; Martina Gahlemann, clinical care, BI, Germany; Luigi Visintin, legal affairs, LIAF, Italy; Hazel Evans, paediatric care, Southampton, UK; Martine Puhl, patient representation (co-chair), NAF, The Netherlands; Lina Buzermaniene, patient representation, EFA, Lithuania; Val Hudson, patient representation, Asthma UK; Laura Bond, patient representation, Asthma UK; Pim de Boer, patient representation and pathobiology, IND; Guy Widdershoven, research ethics, VUMC, The Netherlands; Ralf Sigmund, research methodology and biostatistics, BI, Germany. The patient input platform: Amanda Roberts, UK; David Supple (chair), UK; Dominique Hamerlijnck, The Netherlands; Jenny Negus, UK; Juliëtte Kamphuis, The Netherlands; Lehanne Sergison, UK; Luigi Visintin, Italy; Pim de Boer (co-chair), The Netherlands; Susanne Onstein, The Netherlands. Members of the safety monitoring board: William MacNee, clinical care; Renato Bernardini, clinical pharmacology; Louis Bont, paediatric care and infectious diseases; Per-Ake Wecksell, patient representation; Pim de Boer, patient representation and pathobiology (chair); Martina Gahlemann, patient safety advice and clinical care (co-chair); Ralf Sigmund, bio-informatician.

Author Contributions: K. Takahashi, C. Rossios, M. Loza, F. Ng Kee Kwong, K. Sun and S. Pavlidis performed the analysis; K. Takahashi, K.F. Chung, M. Loza, F. Baribaud, B. de Muelder, C. Auffray, I.M. Adcock and Y. Guo designed the analytica approaches taken and analysed the results; U. Hoda, P. Montuschi, F. Singer, J. Musial, P. Bakke, R. Lutter, P. Chanez, S.J. Fowler, I. Horvath, N. Krug, T. Sandstrom, D.E. Shaw, L.J. Fleming, P.H. Howarth, M. Caruso and B. Dahlen participated in the clinical characterisation of the patients; K. Sun, A.R. Sousa and J. Corfield were part of the data curation team; I.M. Adcock, R. Djukanovic, P.J. Sterk and K.F. Chung conceived of and designed the study; and K. Takahashi, I.M. Adcock and K.F. Chung coordinated the data and drafted the manuscript. All authors read the final version of the manuscript. 
Conflict of interest: K. Takahashi received personal fees from Asahi General Hospital, during the conduct of the study. M. Loza is employed by and owns stock in Johnson \& Johnson, the parent company of Janssen R\&D. F. Baribaud is an employee and shareholder of Janssen R\&D. P. Chanez has provided consultancy services for Boehringer Ingelheim, Johnson \& Johnson, GlaxoSmithKline, Merck Sharp \& Dohme, AstraZeneca, Novartis, Teva, Chiesi, Sanofi and SNCF; has served on advisory boards for Almirall, Boehringer Ingelheim, Johnson \& Johnson, GlaxoSmithKline, AstraZeneca, Novartis, Teva, Chiesi and Sanofi; has received lecture fees from Boehringer Ingelheim, Centocor, GlaxoSmithKline, AstraZeneca, Novartis, Teva, Chiesi, Boston Scientific and ALK; and has received industry-sponsored grants from Roche, Boston Scientific, Boehringer Ingelheim, Centocor, GlaxoSmithKline, AstraZeneca, ALK, Novartis, Teva and Chiesi. I. Horvath has received personal fees from Astra Zeneca, Boehringer Ingelheim, Novartis, CSL, Chiesi, Roche, GSK, Berlin-Chemie and Sandoz, outside the submitted work. P. Montuschi reports personal fees from advisory board meetings with AstraZeneca, outside the submitted work. F. Singer has received personal fees and honoraria for speaking engagements from Vertex and Novartis, outside the submitted work. S-E. Dahlén has received research grants from several Swedish funding bodies such as MRC, Heart-Lung-Foundation and the Strategic Research Foundation, during the conduct of the study; and has received personal fees for advisory board meetings from GSK, AZ, Novartis, Teva, Regeneron/Sanofi, Merck and RSPR AB, grants on asthma phenotyping from AZ, and honoraria for speaking engagements from GSK and Teva, outside the submitted work. B. Dahlén has received personal fees from Teva (for advisory board membership) and AstraZeneca (payments for lectures), outside the submitted work. N. Krug reports grants from IMI (U-BIOPRED consortium IMI number 115010), during the conduct of the study. T. Sandström has received personal fees from advisory board meetings with pharmaceutical companies GSK, AZ, Novartis, Teva and Boehringer Ingelheim, and honoraria for speaking engagements for AZ, Novartis, Boehringer Ingelheim, outside the submitted work. D.E. Shaw has received personal fees for advisory board work from GSK, AZ, Teva and Boehringer Ingelheim, outside the submitted work. R. Lutter has received personal fees for advisory board meetings from GSK and Boehringer Ingelheim, and grants on asthma and COPD from GSK, Lung Foundation and MedImmune, outside the submitted work. P. Bakke has received personal fees for advisory board meetings from GSK, AZ, Novartis and Teva, and honoraria for speaking engagements from AZ and Boehringer Ingelheim, outside the submitted work. L.J. Fleming has received personal fees for advisory board meetings from Novartis, Vectura and Boehringer Ingelheim, grants from Asthma UK and BLF, and honoraria for speaking engagements for Novartis, outside the submitted work. P.H. Howarth was employed part time by GSK as Global Medical Expert. M. Caruso received grants (paid to institute) from the Innovative Medicines Initiative (IMI), during the conduct of the study. C. Auffray received grants from the Innovative Medicine Initiative (U-BIOPRED Consortium IMI number 115010 and eTRIKS Consortium IMI number 115446), during the conduct of the study. B. De Meulder received grants from the Innovative Medicine Initiative (U-BIOPRED Consortium IMI number 115010 and eTRIKS Consortium IMI number 115446), during the conduct of the study. D. Lefaudeux received grants from the Innovative Medicine Initiative (U-BIOPRED Consortium IMI number 115010 and eTRIKS Consortium IMI number 115446), during the conduct of the study. R. Djukanovic has received personal fees for lectures at company sponsored symposia and consulting in advisory boards from TEVA, grants (for an investigator led study of mechanisms of action of omalizumab) and personal fees (for lectures at company sponsored symposia and consulting in advisory boards) from Novartis, and is a consultant to, co-founder of, and holds shares in Synairgen, outside the submitted work. P.J. Sterk has received a public-private grant (paid to institute) from Innovative Medicines Initiative (IMI) covered by the European Union (EU) and the European Federation of Pharmaceutical Industries and Associations (EFPIA), during the conduct of the study. I.M. Adcock has received personal fees for advisory board meetings from GSK, AZ, Novartis, Boehringer Ingelheim and Vectura, grants on asthma and COPD from Pfizer, GSK, MRC, EU, BI and IMI, and honoraria for speaking engagements for AZ and BI, outside the submitted work. K.F. Chung has received personal fees for advisory board meetings from GSK, AZ, Novartis, Teva, Boehringer Ingelheim and J\&J, grants on asthma and COPD from Pfizer, GSK, MRC, EU IMI and NIH, and honoraria for speaking engagements for $\mathrm{AZ}$ and Merck, outside the submitted work.

Support statement: The U-BIOPRED project is supported through an Innovative Medicines Initiative joint undertaking under grant agreement 115010, resources of which are composed of financial contributions from the European Union's Seventh Framework Programme (FP7/2007-2013) and European Federation of Pharmaceutical Industries and Associations companies' in-kind contributions (www.imi.europa.eu). Funding information for this article has been deposited with the Crossref Funder Registry.

\section{References}

1 Chung KF, Wenzel SE, Brozek JL, et al. International ERS/ATS guidelines on definition, evaluation and treatment of severe asthma. Eur Respir J 2014; 43: 343-373.

2 Cerveri I, Cazzoletti L, Corsico AG, et al. The impact of cigarette smoking on asthma: a population-based international cohort study. Int Arch Allergy Immunol 2012; 158: 175-183.

3 Thomson NC, Chaudhuri R. Asthma in smokers: challenges and opportunities. Curr Opinion Pulm Med 2009; 15: $39-45$.

4 Lefaudeux D, De Meulder B, Loza MJ, et al. U-BIOPRED clinical adult asthma clusters linked to a subset of sputum omics. J Allergy Clin Immunol 2017; 139: 1797-1807.

5 Hardin M, Cho M, McDonald ML, et al. The clinical and genetic features of COPD-asthma overlap syndrome. Eur Respir J 2014; 44: 341-350.

6 Wenzel SE. Asthma phenotypes: the evolution from clinical to molecular approaches. Nat Med 2012; 18: 716-725.

7 Shaw DE, Sousa AR, Fowler SJ, et al. Clinical and inflammatory characteristics of the European U-BIOPRED adult severe asthma cohort. Eur Respir J 2015; 46: 1308-1321.

8 Kuo CS, Pavlidis S, Loza M, et al. A transcriptome-driven analysis of epithelial brushings and bronchial biopsies to define asthma phenotypes in U-BIOPRED. Am J Respir Crit Care Med 2017; 195: 443-455.

9 Kuo CS, Pavlidis S, Loza M, et al. T-helper cell type 2 (Th2) and non-Th2 molecular phenotypes of asthma using sputum transcriptomics in U-BIOPRED. Eur Respir J 2017; 49: 1602135.

10 Szklarczyk D, Franceschini A, Wyder S, et al. STRING v10: protein-protein interaction networks, integrated over the tree of life. Nucleic Acids Res 2015; 43: Database issue D447-D452. 
11 Hänzelmann S, Castelo R, Guinney J. GSVA: gene set variation analysis for microarray and RNA-seq data. $B M C$ Bioinformatics 2013; 14: 7.

12 Gasson JC. Molecular physiology of granulocyte-macrophage colony-stimulating factor. Blood 1991; 77: $1131-1145$

13 Schroeder BW, Verhaeghe C, Park SW, et al. AGR2 is induced in asthma and promotes allergen-induced mucin overproduction. Am J Respir Cell Mol Biol 2012; 47: 178-185.

14 McCabe D, Cukierman T, Gabay JE. Basic residues in azurocidin/HBP contribute to both heparin binding and antimicrobial activity. J Biol Chem 2002; 277: 27477-27488.

15 Blatt AZ, Pathan S, Ferreira VP. Properdin: a tightly regulated critical inflammatory modulator. Immunol Rev 2016; 274: 172-190.

16 Hiebert PR, Granville DJ. Granzyme B in injury, inflammation, and repair. Trends Mol Med 2012; 18: 732-741

17 Sanjurjo L, Aran G, Roher N, et al. AIM/CD5L: a key protein in the control of immune homeostasis and inflammatory disease. J Leukocyte Biol 2015; 98: 173-184.

18 Androutsopoulos VP, Tsatsakis AM, Spandidos DA. Cytochrome P450 CYP1A1: wider roles in cancer progression and prevention. BMC Cancer 2009; 9: 187.

19 Gaut JR, Hendershot LM. The modification and assembly of proteins in the endoplasmic reticulum. Curr Opin Cell Biol 1993; 5: 589-595.

20 Osorio F, Lambrecht B, Janssens S. The UPR and lung disease. Semin Immunopathol 2013; 35: 293-306.

21 Spira A, Beane J, Shah V, et al. Effects of cigarette smoke on the human airway epithelial cell transcriptome. Proc Natl Acad Sci USA 2004; 101: 10143-10148.

22 Ludwig A, Petersen F, Zahn S, et al. The CXC-chemokine neutrophil-activating peptide-2 induces two distinct optima of neutrophil chemotaxis by differential interaction with interleukin-8 receptors CXCR-1 and CXCR-2. Blood 1997; 90: 4588-4597.

23 Wang G, Baines KJ, Fu JJ, et al. Sputum mast cell subtypes relate to eosinophilia and corticosteroid response in asthma. Eur Respir J 2016; 47: 1123-1133.

24 Imoto Y, Tokunaga T, Matsumoto Y, et al. Cystatin SN upregulation in patients with seasonal allergic rhinitis. PLoS One 2013; 8: e67057.

25 Hansson GC. Role of mucus layers in gut infection and inflammation. Curr Opin Microbiol 2012; 15: 57-62.

26 Schuijs MJ, Willart MA, Hammad H, et al. Cytokine targets in airway inflammation. Curr Opin Pharmacol 2013; 13: $351-361$

27 Cates EC, Fattouh R, Wattie J, et al. Intranasal exposure of mice to house dust mite elicits allergic airway inflammation via a GM-CSF-mediated mechanism. J Immunol 2004; 173: 6384-6392.

28 Vlahos R, Bozinovski S, Chan SP, et al. Neutralizing granulocyte/macrophage colony-stimulating factor inhibits cigarette smoke-induced lung inflammation. Am J Respir Crit Care Med 2010; 182: 34-40.

29 Botelho FM, Nikota JK, Bauer C, et al. A mouse GM-CSF receptor antibody attenuates neutrophilia in mice exposed to cigarette smoke. Eur Respir J 2011; 38: 285-294.

30 Chung KF, Marwick JA. Molecular mechanisms of oxidative stress in airways and lungs with reference to asthma and chronic obstructive pulmonary disease. Ann NY Acad Sci 2010; 1203: 85-91.

31 Polosa R, Thomson NC. Smoking and asthma: dangerous liaisons. Eur Respir J 2013; 41: 716-726.

32 Aldakheel FM, Thomas PS, Bourke JE, et al. Relationships between adult asthma and oxidative stress markers and pH in exhaled breath condensate: a systematic review. Allergy 2016; 71: 741-757.

33 Kim SR, Kim DI, Kang MR, et al. Endoplasmic reticulum stress influences bronchial asthma pathogenesis by modulating nuclear factor $\mathrm{\kappa B}$ activation. J Allergy Clin Immunol 2013; 132: 1397-1408.

34 Somborac-Bacura A, van der Toorn M, Franciosi L, et al. Cigarette smoke induces endoplasmic reticulum stress response and proteasomal dysfunction in human alveolar epithelial cells. Exp Physiol 2013; 98: 316-325.

35 Titz B, Sewer A, Schneider T, et al. Alterations in the sputum proteome and transcriptome in smokers and early-stage COPD subjects. J Proteomics 2015; 128: 306-320.

36 Gally F, Chu HW, Bowler RP. Cigarette smoke decreases airway epithelial FABP5 expression and promotes Pseudomonas aeruginosa infection. PLoS One 2013; 8: e51784.

37 Schamberger AC, Mise N, Meiners S, et al. Epigenetic mechanisms in COPD: implications for pathogenesis and drug discovery. Expert Opin Drug Discov 2014; 9: 609-628.

38 Overbeek SA, Braber S, Koelink PJ, et al. Cigarette smoke-induced collagen destruction; key to chronic neutrophilic airway inflammation? PLoS One 2013; 8: e55612.

39 Iversen LF, Kastrup JS, Bjørn SE, et al. Structure of HBP, a multifunctional protein with a serine proteinase fold. Nat Struct Biol 1997; 4: 265-268.

40 Haruta I, Kato Y, Hashimoto E, et al. Association of AIM, a novel apoptosis inhibitory factor, with hepatitis via supporting macrophage survival and enhancing phagocytotic function of macrophages. J Biol Chem 2001; 276: 22910-22914.

41 Zhang Q, Cox M, Liang Z, et al. Airway microbiota in severe asthma and relationship to asthma severity and phenotypes. PLoS One 2016; 11: e0152724.

42 Chung KF. Airway microbial dysbiosis in asthmatic patients: a target for prevention and treatment? J Allergy Clin Immunol 2017; 139: 1071-1081. 